\title{
Simultaneous Wireless Information and Power Transfer at 5G New Frequencies: Channel Measurement and Network Design
}

\author{
Daosen Zhai, Member, IEEE, Ruonan Zhang, Member, IEEE, Jianbo Du, \\ Zhiguo Ding, Senior Member, IEEE, and F. Richard Yu, Fellow, IEEE
}

\begin{abstract}
Simultaneous wireless information and power transfer (SWIPT) technique offers a potential solution to ease the contradiction between high data rate and long standby time in the fifth generation (5G) mobile communication systems. In this paper, we focus on the SWIPT network design and optimization with 5G new frequencies. To design an efficient SWIPT network, we first investigate the propagation properties of 5G low-frequency (LF) and high-frequency (HF) channels. Specifically, a measurement campaign focusing on $3.5 \mathrm{GHz}$ and $28 \mathrm{GHz}$ is conducted in both outdoor and outdoor-to-indoor scenarios. Motivated by the measurement results, we design a dual-band SWIPT network, where the HF band is used for short-distance information delivery, while the LF band is used for short-distance energy transfer and long-distance information delivery. The designed network has a win-win architecture that can enhance the throughput of cell-edge users and improve the energy-harvesting efficiency of cell-center users. To further boost the network performance, we devise a joint power-and-channel allocation algorithm, which has the advantages of low complexity and fast convergence. Finally, simulation results demonstrate that the designed dual-band network outperforms the conventional single-band network in terms of energy-harvesting efficiency and user fairness, and the proposed algorithm can further upgrade the network performance significantly.
\end{abstract}

Index Terms-Simultaneous wireless information and power transfer, 5G new frequency, channel measurement, network design, resource allocation.

\section{INTRODUCTION}

With the evolution of communication technologies, the fifth generation $(5 \mathrm{G})$ of mobile communications is expected to be commercialized towards year 2020 and beyond. As a great improvement compared with previous generation systems, $5 \mathrm{G}$ can provide users with Gbps user experienced data rate and almost zero end-to-end latency, such that some new applications as ultra-high-definition video, mobile cloud, and virtual reality can be supported even under the moving state [1]. In addition to upgrading the quality-of-service (QoS) of subscribers, improving the quality-of-experience $(\mathrm{QoE})$ is also a major concern in $5 \mathrm{G}$ wireless networks [2]. However, the

Daosen Zhai and Ruonan Zhang are with School of Electronics and Information, Northwestern Polytechnical University, Xi'an, Shaanxi, 710072, China (e-mail: zhaidaosen@nwpu.edu.cn; rzhang@nwpu.edu.cn).

Jianbo Du is with the State Key Laboratory of Integrated Service Networks, Xidian University, Xi'an 710071, China (e-mail: dujianboo@163.com).

Zhiguo Ding is also with the School of Computing and Communications, Lancaster University, Lancaster, UK (email: z.ding@lancaster.ac.uk).

F. Richard $\mathrm{Yu}$ is with the Department of Systems and Computer Engineering, Carleton University, Ottawa, ON K1S 5B6, Canada (e-mail: richard.yu@carleton.ca). improvement of data rate usually causes higher energy consumption, which degrades the $\mathrm{QoE}$ of the users with batterypowered devices. Furthermore, some Internet of Things (IoT) equipments such as the wearable devices and sensor nodes are more sensitive to energy deficit, as recharging these devices is inconvenient and sometimes infeasible. Therefore, how to deal with the contradiction between energy conservation and rate improvement has become a prominent issue in the design and optimization of $5 \mathrm{G}$ wireless networks.

As a potential solution, wireless power transfer (WPT) technique can be employed to recharge mobile devices through radio frequency (RF) signals [3]. Different from the fluctuant energy sources in environment (e.g., solar and wind energy), the RF signals can be controlled so as to provide a stable and reliable energy supply. To facilitate the implementation of WPT, some researchers suggest to deploy some power beacons on the basis of the existing networks [4]. However, deploying power beacons leads to additional constructing and operating expenditure that is cost-inefficient. As such, it is more advisable to exploit the existing communication base stations (BSs) for WPT, which results in the paradigm of simultaneous wireless information and power transfer (SWIPT). The concept of SWIPT was first conceived in [5], where the authors made an ideal assumption that the received radio signals can be simultaneously utilized for energy harvesting (EH) and information decoding (ID). Considering the limitation of realistic receivers, the authors in [6] proposed two rational energy-harvesting approaches, namely the powersplitting (PS) approach and the time-switching (TS) approach. With the PS and TS approaches, the processes of EH and ID are separated in the power and time domains, respectively. As a further study, the circuit design of these two approaches was investigated in [7]. Additionally, if the energy harvester is equipped with multi-receivers or multi-antennas, the separated receiver architecture or the antenna-switching scheme can also be utilized for SWIPT [8].

In order to take full use of the SWIPT technique, abundant researches have been conducted on how to incorporate it with other communication technologies. Compared with the nearfield point-to-point WPT, the RF SWIPT has the benefit of powering multiple users in a long distance. Due to this reason, the primary application scenarios of SWIPT were focused on the multiple access (MA) networks, such as the time-division multiple access (TDMA) [9], orthogonal frequency-division multiple access (OFDMA) [10], [11], and non-orthogonal mul- 
tiple access (NOMA) networks [12], [13]. In these networks, appropriate resource management is the key problem, as it directly determines the tradeoff between the data transmission rate and energy-harvesting rate. Specifically, the authors in [9] proposed an order-based equal throughput fair scheduler, which can provide users with proportionally fair energyharvesting rate. Different from [9], the authors in [10] and [11] concentrated on the multi-carrier networks and investigated the joint power-and-channel allocation algorithms for sum-rate maximization and energy-efficiency promotion, respectively. Except for the orthogonal MA techniques, NOMA is also a candidate technique in $5 \mathrm{G}$ wireless networks [12], [13]. In NOMA networks, the diversity of users in the power domain is exploited, such that more users can be supported on the same resource blocks. The non-orthogonal feature of NOMA is also in favor of energy transfer. In sparse code multiple access (SCMA) networks, an iterative resource allocation algorithm was devised in [12] to maximize the weighted sum rate and energy. It shows that SCMA networks can achieve a better rate-energy tradeoff with respect to OFDMA networks. In [13], a cooperative relay protocol combined with SWIPT and NOMA was proposed to enhance the throughput of cell-edge users. Particularly, the near NOMA users close to the source can harvest energy and then use the harvested energy to relay data for the far NOMA users. With the advance of research, the application scenarios of SWIPT were extended to the multipleinput multiple-output (MIMO) systems [14]-[16], cognitive radio networks [17], [18], and sensor networks [19]-[21]. As a survey, [22] summarized the recent research advancements of SWIPT.

As a common feature, the above works [9]-[21] focus on the networks with conventional low-frequency (LF) band. However, the bandwidth in LF band is very limited, which cannot satisfy the ultra-high rate requirement of $5 \mathrm{G}$. In this circumstance, exploiting the high-frequency (HF) band, i.e., millimeter wave (mmWave), becomes an inevitable choice for increasing bandwidth. Recently, the SWIPT assisted mmWave communication has become a hot topic. In [23], the successful design of the rectenna at $24 \mathrm{GHz}$ demonstrated the feasibility of WPT towards mmWave regime. Besides, a harvest-anduse strategy was proposed in [24], where the devices can harvest energy from the BS through mmWave signals and then use the harvested energy to transmit data. Adopting the stochastic geometry approach, the authors in [25] analyzed the energy coverage probability of mmWave networks with multi-antenna arrays. The simulation results in [25] indicated that mmWave combined with multi-antenna arrays generally outperforms LF solutions in terms of energy-harvesting rate. The authors in [26] investigated the performance of WPT in mmWave massive MIMO systems operating in rainy or clear conditions. It demonstrated that the rain attenuation has a great effect on the energy-harvesting efficiency in HF band and can even make the WPT impossible in the severe case. The performance of relay-aided mmWave massive MIMO system was analyzed in [27]. In the similar system, [28] investigated the energy-harvesting potential of mmWave based on the stochastic geometry theory. All works in [23]-[28] express that mmWave can be utilized for WPT, but it must be combined with multiple antennas. Besides, the WPT through mmWave is more susceptible to environmental effects, such as the obstruction and rain attenuation.

Although the existing researches have promoted the development of SWIPT, there are still some open issues remained to be tackled. Firstly, all researches in [9]-[21], [23]-[28] only focus on the single-band system. However, as indicated in the 5G white paper on technology architecture [29], all-spectrum access involving LF and HF bands has been recommended as one of the $5 \mathrm{G}$ key technologies. Unfortunately, none of researches pay attention to the SWIPT issues in the networks with hybrid bands, which deserves much research. Furthermore, the works in [9]-[21], [23]-[28] take into account the existing channel models without experimental verification, and hence the accuracy of the simulation results needs to be further investigated. Moreover, some new frequencies such as $3.5 \mathrm{GHz}$ in $\mathrm{LF}$ band and $28 \mathrm{GHz}$ in $\mathrm{HF}$ band will be utilized in $5 \mathrm{G}$ wireless networks, the channel characteristics of which are totally different from the conventional LF channels below 3 $\mathrm{GHz}$ [30], [31]. Since either power transfer or information delivery is highly dependent on the channel characteristics, it is thus necessary to implement channel measurement for $5 \mathrm{G}$ new frequencies in order to acquire the channel propagation properties. Besides, as a fringe benefit, the measurement results can also provide some valuable guidances for the design and optimization of the SWIPT networks.

Motivated by the above, we systematically investigate the SWIPT problems with $5 \mathrm{G}$ new frequencies, including the channel measurement, network design, and network optimization. The contributions of this paper are summarized as follows.

- We measure the large-scale fading of the channels at 3.5 $\mathrm{GHz}$ and $28 \mathrm{GHz}$. Specifically, a high-precision dualband channel sounder is adopted to conduct the measurement campaign. Specifically, we choose the typical urban roads and a corridor in a U-type building to emulate the outdoor and outdoor-to-indoor communication scenes, respectively. The measurement results indicate that the HF band is only suitable for short-distance information delivery, while the LF band is suitable for short-distance power transfer and long-distance information delivery. In addition, a more accurate channel model especially for $3.5 \mathrm{GHz}$ and $28 \mathrm{GHz}$ is constructed based on the plenty of measurement data, which offers a more precise design guidance for the SWIPT networks with 5G new frequencies.

- Motivated by the measurement results, we design a dualband SWIPT network with two different coverage region$\mathrm{s}$, referred to as the hot-spot region (HSR) and widearea coverage region (WCR). In particular, the WCR is responsible for seamless coverage, in which the devices can only receive information from the LF band. While in the HSR, the devices can receive information from the HF band and harvest energy from the LF band through the TS energy-harvesting approach. It is worthy to point out that the energy harvested by the devices in the HSR derives from the RF signals of the users in the WCR. As such, by allocating more power on the LF band, the achievable 
data rate of the cell-edge users is enhanced, and meanwhile the energy-harvesting efficiency of the cell-center users is also improved. Therefore, our designed network has a win-win architecture.

- To further upgrade the performance of the designed network, we formulate a joint power-and-channel allocation problem, with the objective to maximize the minimum harvested energy of the users in the HSR. Based on the dual decomposition technique and the matching theory, an iterative algorithm is proposed to solve the formulated problem in a low complexity manner. To demonstrate the advantages of the designed network and algorithm, we compare them with other schemes via simulations. The simulation results indicate that the dual-band network outperforms the single-band network in terms of energyharvesting efficiency and user fairness even with the random resource allocation. Furthermore, incorporated with our proposed resource allocation algorithm, the performance of the dual-band network can be further enhanced significantly.

The remainder of this paper is organized as follows. In section II, we demonstrate the channel measurement and modeling. Section III introduces the designed network and the problem formulation. In section IV, we elaborate the proposed resource allocation algorithm. Simulation results are presented in section V. Finally, we conclude our paper in Section VI.

\section{Channel Measurement and Modeling}

In this section, we first introduce the channel measurement system and scenario, followed by presenting the measurement results and some analysis.

\section{A. Measurement System}

To evaluate the feasibility of SWIPT with 5G new frequencies, we focus on the large-scale fading at $3.5 \mathrm{GHz}$ and $28 \mathrm{GHz}$. As shown in Fig. 1, we adopt a dual-band large-scale channel sounder, developed jointly by Huawei and Northwestern Polytechnical University.

On the the transmitter (Tx) side, two RF chains are installed to transmit the single tones at the target carrier frequencies, i.e., $3.5 \mathrm{GHz}$ and $28 \mathrm{GHz}$. Each $\mathrm{RF}$ chain is composed of a signal generator, power amplifier, and transmitting antenna. As depicted in Fig. 1 (a), the horn antenna on the left is used for radiating $28 \mathrm{GHz}$ probe signals, which is generated by the signal generator N5183A. The horn antenna is directional with the maximum gain of $8.5 \mathrm{dBi}$. The half power beam width (HPBW) of the antenna are $100^{\circ}$ in the azimuth plane and $40^{\circ}$ in the elevation plane. The power amplifier working at 28 $\mathrm{GHz}$ has a gain of $63 \mathrm{dBm}$. In addition, the white cylindrical antenna on the right radiates $3.5 \mathrm{GHz}$ probe signals, which is generated by the signal generator E4438C. This antenna is omni-directional in the azimuth plane and features $30^{\circ} \mathrm{HPBW}$ in the elevation plane with a gain of $4 \mathrm{dBi}$. The relevant power amplifier has a gain of $43 \mathrm{dBm}$.

The receiver (Rx) shown in Fig. 1 (b) is equipped with a wideband antenna, low noise amplifier (LNA), and portable spectrum analyzer (SA), which can capture and analyze the

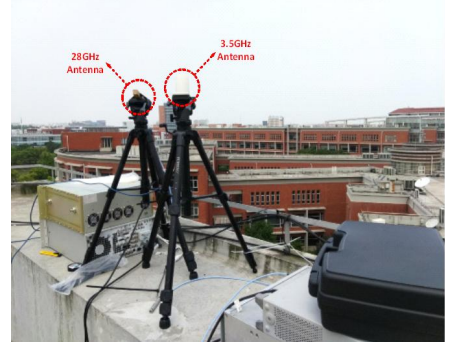

(a) Transmitter

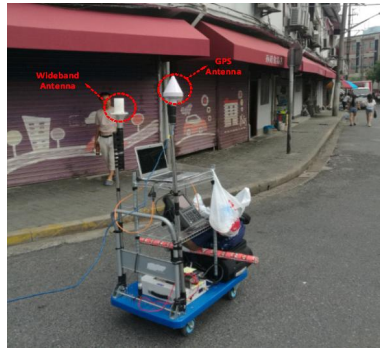

(b) Receiver
Fig. 1. Measurement system with a dual-band transmitter and a wide-band receiver.

probe signals at both frequencies. The receiving antenna is omni-directional with the gain of $4 \mathrm{dBi}$ and $2.5 \mathrm{dBi}$ at $3.5 \mathrm{GHz}$ and $28 \mathrm{GHz}$, respectively. The received signal is first amplified by the LNA and then input into the SA. The function of the SA is to measure the power level of the received signal. To eliminate the effect of small-scale fading, the SA continuously measures 50 power samples of the received signals in each marked position with the interval of $200 \mathrm{~ms}$ and thereby get the average attenuation.

According to the measurement results and system parameters, we can get the average attenuation at $3.5 \mathrm{GHz}$ and 28 $\mathrm{GHz}$ via the formula in (1), where $P_{T X}$ denotes the transmission power, $G_{T X}\left(f_{c}\right)$ denotes the transmitting antenna gain, $G_{R X}\left(f_{c}\right)$ denotes the receiving antenna gain, $G_{L N A}$ denotes the LNA gain, and $P_{R X}$ denotes the received power. Besides, $f_{c}$ is the carrier frequency and $d_{3 D}$ is the 3-dimensional (3D) distance between the Tx and Rx which can be calculated with the aid of the GPS antenna.

$$
P L\left(f_{c}, d_{3 D}\right)=P_{T X}+G_{T X}\left(f_{c}\right)+G_{R X}\left(f_{c}\right)+G_{L N A}-P_{R X} .
$$

\section{B. Measurement Scenario}

The measurement campaign was conducted in both outdoor and outdoor-to-indoor (O2I) scenarios, which are depicted in Fig. 2.

The outdoor scenario was selected as the typical urban roads in Shanghai, China, the satellite map of which is shown in Fig. 2 (a). The Tx was installed on the top of a ten-story building, the height of which is about 30 meters. The Rx was moved along different routes to emulate the line-of-sight (LOS) and non-line-of-sight (NLOS) scenarios. As shown in Fig. 2 (a), we choose 380 measurement positions along the routes to receive the probe signals, thereby evaluating the large-scale fading. The 3D distance between the Tx and Rx ranges from 200 meters to 1000 meters.

On the other hand, we choose a building in Shanghai Jiao Tong University, a U-type corridor in the second floor, to emulate the O2I scenario. The structure diagram of the corridor is shown in Fig. 2 (b), corresponding to the realistic scene shown in Fig. 1 (a). The Tx was installed on the top of another building on the opposite side of the corridor. The $\mathrm{Rx}$ was moved along the corridor, in which 60 measurement positions were marked to capture and analyze the probe 


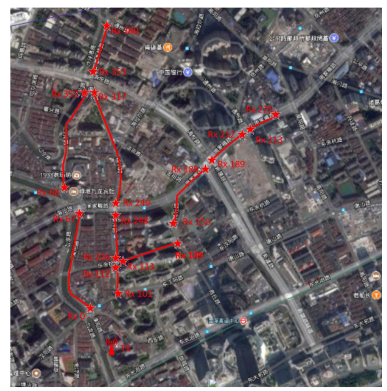

(a) Outdoor Scenario

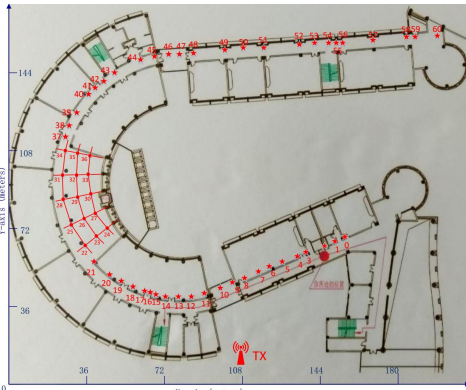

(b) Outdoor-to-Indoor Scenario
Fig. 2. Measurement scenario with a critical urban outdoor scene and an out-to-indoor scene.

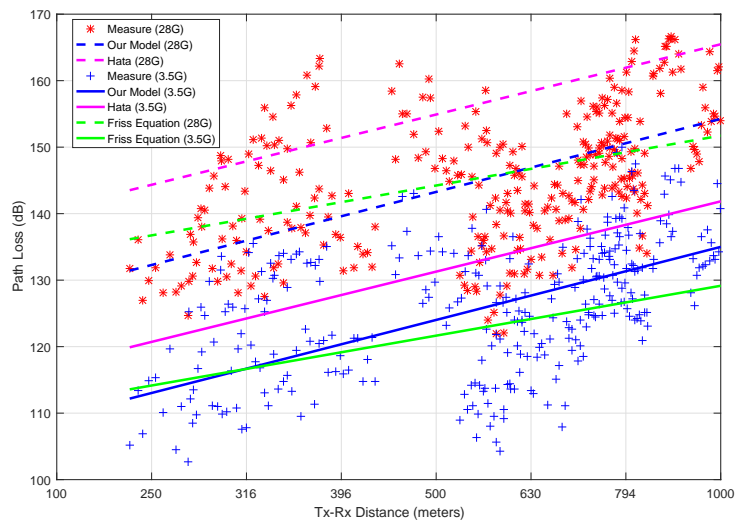

Fig. 3. Measurement results and channel model of the large-scale fading in a typical urban outdoor scenario.

signals. Since there exists abundant windows and walls in the corridor, this scenario can be utilized to well evaluate the penetration loss across different barriers.

\section{Measurement Results and Channel Modeling}

The measurement results in the outdoor scenario are plotted in Fig. 3. From this figure, we can observe that the large-scale fading fluctuates heavily in different positions. This is because there are many buildings in the surrounding environment, which results in complicated reflection and absorption. As a consequence, the signals in some far positions with LOS rays may surpass those in some near positions but only with NLOS rays. Overall, the large-scale fading at $3.5 \mathrm{GHz}$ is much less that that at $28 \mathrm{GHz}$. When the Tx-Rx distance is larger than 316 meters, the large-scale fading at $28 \mathrm{GHz}$ has been larger than $140 \mathrm{~dB}$ in more than half of the marked positions, that is, the HF band has been not suitable for general communication systems over this distance. On the contrary, the $3.5 \mathrm{GHz}$ band can facilitate efficient coverage even when the Tx-Rx distance is 1000 meters.

Based on the measurement results, we construct the largescale fading channel model for $3.5 \mathrm{GHz}$ and $28 \mathrm{GHz}$ by using the least-squares curve fitting method. Specifically, the constructed channel model is given in (2). To illustrate the accuracy of our model, we compare it with the Hata model

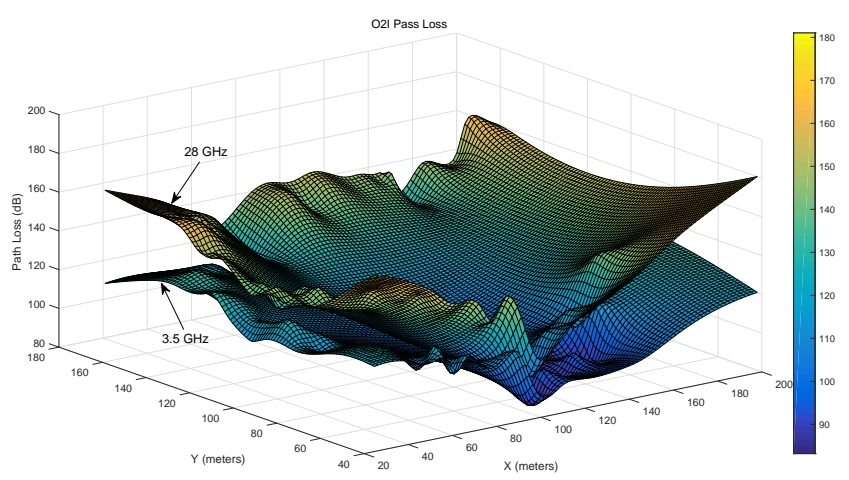

Fig. 4. Measurement results of the large-scale fading in a typical outdoorto-indoor scenario.

[32] and the Friis Equation [33], [34]. To match the measurement results, the pass loss exponent in the Friis Equation is set as 2.5. From Fig. 3, we can observe that the Hata model overestimates the large-scale fading of $5 \mathrm{G}$ new frequencies, especially for $28 \mathrm{GHz}$. Compared with the Hata model, the Friis Equation can better match the measurement results. However, the estimation error grows with the increment of the Tx-Rx distance. As can bee seen, the Friis Equation cannot well model the large-scale fading when the Tx-Rx distance is larger than 800 meters. By contrast, our model can provide an accurate estimation of the large-scale fading at $5 \mathrm{G}$ new frequencies with any $\mathrm{Tx}-\mathrm{Rx}$ distance. Besides, according to the constructed channel model, we can calculate that the largescale fading at $28 \mathrm{GHz}$ is $19 \mathrm{~dB}$ larger than that at $3.5 \mathrm{GHz}$ in a typical urban outdoor scenario.

$$
\begin{aligned}
P L\left(f_{c}, d_{3 D}\right)= & 13.54+21.32 \times \log _{10}\left(f_{c}[\mathrm{GHz}]\right) \\
& +36.62 \times \log _{10}\left(d_{3 D}[\text { meters }]\right) .
\end{aligned}
$$

Fig. 4 presents the measurement results in the O2I scenario. Noted that only 60 positions in Fig. 2 (b) was measured, thus there are only 60 realistic values in Fig. 4. The remainder values are obtained by interpolation in order to give an intuitive sense for the large-scale fading at $5 \mathrm{G}$ new frequencies. This figure shows that the large-scale fading is not fat, as some windows exist along the corridor at certain intervals. Comparing the measurement results in Fig. 3 and Fig. 4, we can find that the attenuation gap between the two frequencies in the O2I scenario is generally larger than that in the outdoor scenario. Besides, the measurement results show that the attenuation across barriers at $28 \mathrm{GHz}$ is very large. This result demonstrates that it is difficult to satisfy the O2I coverage requirement only by the HF band.

For clearer comparison, we choose 4 representative positions shown in Fig. 2 (b) and summarize the results in Table I. As illustrated in the table, the path loss gap between the two frequencies is only about $12.5 \mathrm{~dB}$ in the LOS scene. Owing to the abundant bandwidth, the frequency of $28 \mathrm{GHz}$ is suitable for short-distance information delivery. However, the path loss gap between the two frequencies increases through obstacles and is up to $27 \mathrm{~dB}$ when crossing double walls. The maximal tolerable fading of a traditional communication 
TABLE I

LARGE-SCALE FADING IN TYPICAL O2I SCENES

\begin{tabular}{|c|c|c|c|c|}
\hline Typical scenes & LOS & Single glass & Single wall & Double walls \\
\hline Positions in Fig. 2 (b) & Rx 10 & Rx 5 & Rx 0 & Rx 15 \\
\hline Pass loss at 3.5 GHz (dB) & 87.623 & 90.996 & 115.734 & 119.246 \\
\hline Pass loss at 28 GHz (dB) & 100.123 & 107.427 & 140.262 & 146.341 \\
\hline
\end{tabular}

system is $140 \mathrm{~dB}$. As shown in Table I, the frequency of 28 $\mathrm{GHz}$ can only satisfy the path loss requirement in the O2I scene with single glass, while the frequency of $3.5 \mathrm{GHz}$ can ensure good coverage even in the O2I scene with double walls.

According to the measurement results, we can conclude that the LF band has the characteristics of low path loss and through-wall loss, therefore this band is suitable for long-distance information delivery and short-distance energy transfer. On the contrary, the RF signals in HF band undergo large attenuation especially through obstacles. However, the bandwidth of HF band is abundant, such that this band is suitable for short-distance information delivery. In light of these, we design a dual-band SWIPT network, which is specified in the following section.

Finally, we highlight the contributions of our experimentation, which are summarized as the following three points.

1) Although the general propagation properties of the LF and HF channels are well-known, their specific propagation parameters are still undetermined, especially for the 5G new frequencies. Our channel measurement campaign is conducted based on a high-precision dual-band sounder, developed jointly by Huawei and Northwestern Polytechnical University. The measurement results can provide an accurate depiction for the wireless channels of $5 \mathrm{G}$ new frequencies, which is vital for practical network design and optimization.

2) The measurement results can provide useful design guidance for practical SWIPT system. Although the dual-band SWIPT network architecture is proposed based on the general propagation properties of the LF and HF channels, more specific system parameters must be configured according to the accurate channel parameters. For instance, we can calculate the maximum energy transmission distance and the maximum communication distance according to our constructed channel model, thereby defining the coverage regions of the dualband network. In addition, the power transmitter and power harvester should also be designed in accordance with the channel propagation parameters, so as to construct a stable and efficient SWIPT system.

3) Based on the experimental data, we propose a more accurate channel model especially for $3.5 \mathrm{GHz}$ and $28 \mathrm{GHz}$. With this channel model, we can conduct simulations to evaluate the performance of the designed dual-band SWIPT network as well as the proposed joint power-and-channel allocation algorithm. The simulation results based on an accurate channel model are more convincing.

\section{Network Design For SWIPT With 5G NeW FREQUENCIES}

Motivated by the measurement results, we in this section design a dual-band wireless network for SWIPT at 5G new

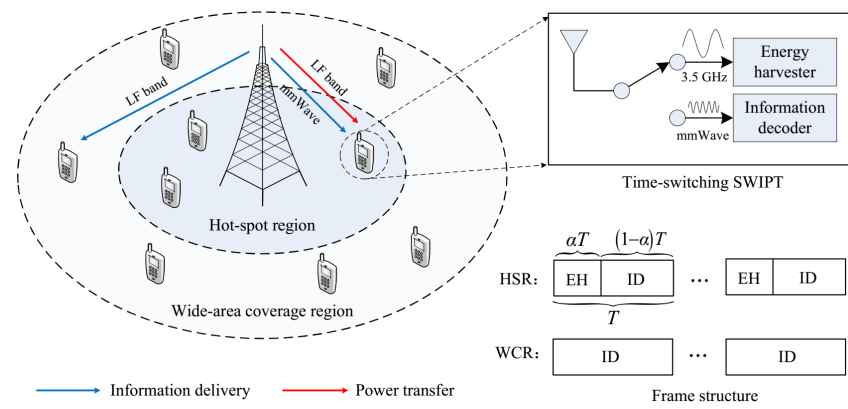

Fig. 5. Dual-band SWIPT network with 5G new frequencies: architecture and frame structure.

frequencies. To further enhance the network performance, a joint power-and-channel allocation problem is formulated.

\section{A. Network Design}

As shown in Fig. 5, the coverage area of the designed SWIPT network is divided into two regions ${ }^{1}$, namely the hot-spot region (HSR) and the wide-area coverage region (WCR). The users located in the HSR are denoted by $\mathcal{K}_{1}=$ $\left\{k_{1} \mid k_{1}=1,2, \cdots, K_{1}\right\}$. Since these users are adjacent to the $\mathrm{BS}$, we assume that they can receive information from the BS via mmWave and meanwhile harvest energy through the LF band. The users located in the WCR are denoted by $\mathcal{K}_{2}=\left\{k_{2} \mid k_{2}=1,2, \cdots, K_{2}\right\}$, wherein each user can only receive information from the BS through the LF band. Note that the LF band is utilized for both energy transfer for the users in the HSR and information delivery for the users in the WCR. As such, improving the transmission power on the LF band is beneficial for enhancing the throughput of the cell-edge users as well as boosting the energy-harvesting rate

\footnotetext{
${ }^{1}$ The criterion for defining two regions is mainly dependent on the receiver sensitivity of the power harvester and the information decoder. In practical system, if the power level of the radio waves is too low, the power harvester cannot capture the RF power and thereby cannot convert the radio waves to directional current (DC) power. Given a power transmitter and a power harvester, there must be a maximum energy transmission distance (METD), as the maximum communication distance (MCD) of the BS. For instance, the receiver sensitivity of the POWERCAST PCC110/PCC210, a commercial product of wireless power transfer, is $-17 \mathrm{dBm}$ [35]. If the power transmitter is a traditional BS with 40-watt maximum transmission power, the channel model is given in (2) with $3.5 \mathrm{GHz}$ carrier frequency, and the antenna gain is $12 \mathrm{dBi}$, it can be calculated that the METD is about 23 meters for the POWERCAST PCC110/PCC210. In academic researches, some high-sensitive power harvesters are designed and tested, the receiver sensitivity of which can be lower to $-39 \mathrm{dBm}$ [3]. In the same scenario, the METD of these harvesters is about 90 meters. For a communication system with $-60 \mathrm{dBm}$ receiver sensitivity, it can be calculated that the MCD at $28 \mathrm{GHz}$ band is about 100 meters, while the MCD at $3.5 \mathrm{GHz}$ band is about 350 meters. Since the METD at LF band and the MCD at HF band are very similar, we combine them into the same region named as the HSR, while the MCD at LF band is relatively large and thus defined as another region named as the WCR.
} 
of the cell-center users. Therefore, our designed dual-band SWIPT network has a win-win architecture. Furthermore, the time-switching approach ${ }^{2}$ is adopted for the users in the HSR to realize information decoding (ID) and energy harvesting (EH), as depicted in Fig. 5. In particular, we set a fixed time-switching ratio ${ }^{3}$, that is, the time $\alpha T$ is used for $\mathrm{EH}$ and the remainder time $(1-\alpha) T$ is used for ID, where $T$ represents an operational period. Without loss of generality, $T$ is normalized to one in this paper.

There are $N_{1}$ channels in the $\mathrm{HF}$ band and $N_{2}$ channels in the LF band, which are denoted by $\mathcal{N}_{1}=$ $\left\{n_{1} \mid n_{1}=1,2, \cdots, N_{1}\right\}$ and $\mathcal{N}_{2}=\left\{n_{2} \mid n_{2}=1,2, \cdots, N_{2}\right\}$, respectively. The bandwidth of the HF and LF channels are $B_{1} \mathrm{~Hz}$ and $B_{2} \mathrm{~Hz}\left(B_{1}>B_{2}\right)$, respectively. Besides, the channel assignment indicators are defined as $\mathbf{X}_{1}=\left\{x_{n_{1}, k_{1}} \mid n_{1} \in \mathcal{N}_{1}, k_{1} \in \mathcal{K}_{1}\right\}$ and $\mathbf{X}_{2}=$ $\left\{x_{n_{2}, k_{2}} \mid n_{2} \in \mathcal{N}_{2}, k_{2} \in \mathcal{K}_{2}\right\}$, where $x_{n_{1}, k_{1}}=1$ (or $x_{n_{2}, k_{2}}=1$ ) represents channel $n_{1}$ (or $n_{2}$ ) is assigned to user $k_{1}$ (or $k_{2}$ ), and $x_{n_{1}, k_{1}}=0$ (or $x_{n_{2}, k_{2}}=0$ ) otherwise. In addition, we denote $\mathbf{P}_{1}=\left\{p_{n_{1}, k_{1}} \mid n_{1} \in \mathcal{N}_{1}, k_{1} \in \mathcal{K}_{1}\right\}$ and $\mathbf{P}_{2}=$ $\left\{p_{n_{2}, k_{2}} \mid n_{2} \in \mathcal{N}_{2}, k_{2} \in \mathcal{K}_{2}\right\}$ as the power allocation policies for the users in the HSR and WCR, respectively. In detail, $p_{n_{1}, k_{1}}$ (or $p_{n_{2}, k_{2}}$ ) represents the transmission power allocated for user $k_{1}$ (or $k_{2}$ ) on channel $n_{1}$ (or $n_{2}$ ). For simplicity, we denote $\mathbf{X}=\left\{\mathbf{X}_{1}, \mathbf{X}_{2}\right\}$ and $\mathbf{P}=\left\{\mathbf{P}_{1}, \mathbf{P}_{2}\right\}$.

Given the resource allocation policy $\left\{\mathbf{P}_{1}, \mathbf{X}_{1}, \mathbf{P}_{2}, \mathbf{X}_{2}\right\}$, the achievable data rate of users $k_{1}$ and $k_{2}$ can be respectively expressed as

$$
\begin{gathered}
R_{k_{1}}=(1-\alpha) \sum_{n_{1} \in \mathcal{N}_{1}} x_{n_{1}, k_{1}} B_{1} \log _{2}\left(1+\frac{p_{n_{1}, k_{1}} g_{n_{1}, k_{1}}}{\sigma^{2}}\right), \\
R_{k_{2}}=\sum_{n_{2} \in \mathcal{N}_{2}} x_{n_{2}, k_{2}} B_{2} \log _{2}\left(1+\frac{p_{n_{2}, k_{2}} g_{n_{2}, k_{2}}}{\sigma^{2}}\right),
\end{gathered}
$$

where $g_{n_{1}, k_{1}}$ (or $g_{n_{2}, k_{2}}$ ) represents the channel power gain from the BS to user $k_{1}$ (or $k_{2}$ ) on channel $n_{1}$ (or $n_{2}$ ), and $\sigma^{2}$ is the noise power.

According to the energy-harvesting approach, the energy harvested by user $k_{1}$ in an operational period is given by

$$
E_{k_{1}}=\alpha \sum_{n_{2} \in \mathcal{N}_{2}} g_{n_{2}, k_{1}}\left(\sum_{k_{2} \in \mathcal{K}_{2}} p_{n_{2}, k_{2}} x_{n_{2}, k_{2}}\right) \text {, }
$$

where $g_{n_{2}, k_{1}}$ denotes the channel power gain from the BS to user $k_{1}$ on channel $n_{2}$.

\footnotetext{
${ }^{2}$ In this network, we adopt the time-switching (TS) approach rather than the power-splitting (PS) approach due to the reason that the PS approach deteriorates the signal-to-noise ratio (SNR) of users. As the RF signals at mmWave undergo large attenuation, utilizing the PS approach will greatly affect the information decoding of the users in the HSR. In contrast, the TS approach only decreases the duration of information transmission but without effect on the SNR of users.

${ }^{3}$ The dynamic time-switching approach complicates the system design especially for the frame structure. To simplify the system design, we consider a fixed time-switching ratio $\alpha$ for the SWIPT network. However, the effect of $\alpha$ on the energy-harvesting efficiency is investigated in the simulation studies.
}

Additionally, the total transmission power of the BS is

$$
\begin{aligned}
P^{\text {tot }}= & (1-\alpha) \sum_{n_{1} \in \mathcal{N}_{1}} \sum_{k_{1} \in \mathcal{K}_{1}} p_{n_{1}, k_{1}} x_{n_{1}, k_{1}} \\
& +\sum_{n_{2} \in \mathcal{N}_{2}} \sum_{k_{2} \in \mathcal{K}_{2}} p_{n_{2}, k_{2}} x_{n_{2}, k_{2}} .
\end{aligned}
$$

\section{B. Problem Formulation}

In order to boost the energy-harvesting efficiency of the designed network, we jointly optimize the power allocation and channel assignment for all users in the network. For the sake of fairness, a max-min utility function is adopted as the objective function. Specifically, the joint power-and-channel allocation problem is formulated as

$$
\begin{aligned}
& \max _{\mathbf{P}, \mathbf{X}} \min _{\mathbf{k}_{1} \in \mathcal{K}_{1}} E_{k_{1}} \\
& \text { s.t. } \mathrm{C} 1: P^{\text {tot }} \leq P^{\mathrm{max}} \\
& \mathrm{C} 2: R_{k_{1}} \geq R_{k_{1}}^{\text {req }}, \forall k_{1} \in \mathcal{K}_{1} \\
& \mathrm{C} 3: R_{k_{2}} \geq R_{k_{2}}^{\text {req }}, \forall k_{2} \in \mathcal{K}_{2} \\
& \mathrm{C} 4: \sum_{n_{1} \in \mathcal{N}_{1}} x_{n_{1}, k_{1}}=1, \forall k_{1} \in \mathcal{K}_{1} \\
& \mathrm{C} 5: \sum_{n_{2} \in \mathcal{N}_{2}} x_{n_{2}, k_{2}}=1, \forall k_{2} \in \mathcal{K}_{2} \\
& \mathrm{C} 6: \sum_{k_{1} \in \mathcal{K}_{1}} x_{n_{1}, k_{1}} \leq 1, \forall n_{1} \in \mathcal{N}_{1} \\
& \mathrm{C} 7: \sum_{k_{2} \in \mathcal{K}_{2}} x_{n_{2}, k_{2}} \leq 1, \forall n_{2} \in \mathcal{N}_{2} \\
& \mathrm{C} 8: x_{n_{1}, k_{1}}, x_{n_{2}, k_{2}} \in\{0,1\}, \forall n_{1}, n_{2}, k_{1}, k_{2} \\
& \mathrm{C} 9: p_{n_{1}, k_{1}}, p_{n_{2}, k_{2}} \geq 0, \forall n_{1}, n_{2}, k_{1}, k_{2} .
\end{aligned}
$$

The objective of the formulated problem is to maximize the minimum harvested energy of the users in the HSR, subject to the power constraint $\mathrm{C} 1$, rate constraints $\mathrm{C} 2-\mathrm{C} 3$, and resource allocation variables constraints C4-C9. More detailedly, constraint $\mathrm{C} 1$ limits the maximum transmission power of the BS, which is imposed by the hardware limitation or standard regulation. Constraints $\mathrm{C} 2$ and $\mathrm{C} 3$ specify the minimum rate requirement of the users in $\mathcal{K}_{1}$ and $\mathcal{K}_{2}$, respectively. Furthermore, C4-C8 are the constraints for the channel assignment variables, wherein $\mathrm{C} 4$ and $\mathrm{C} 5$ indicate that each user can only occupy one channel, and C6-C8 together denote that channels are exclusively allocated among users.

\section{Network Optimization Algorithm Design}

In this section, we devise an cost-efficient algorithm based on the matching theory and the dual decomposition technique to solve the formulated problem in (7).

\section{A. Problem Decomposition}

As can be seen in (7), the objective function only depends on the resource allocation scheme of the users in $\mathcal{K}_{2}$, i.e., $\left\{\mathbf{P}_{2}, \mathbf{X}_{2}\right\}$. However, $\mathbf{P}_{1}$ and $\mathbf{P}_{2}$ are coupled together due to the total power constraint $\mathrm{C} 1$. Reducing the transmission power allocated for the users in $\mathcal{K}_{1}$ is in favor of improving 


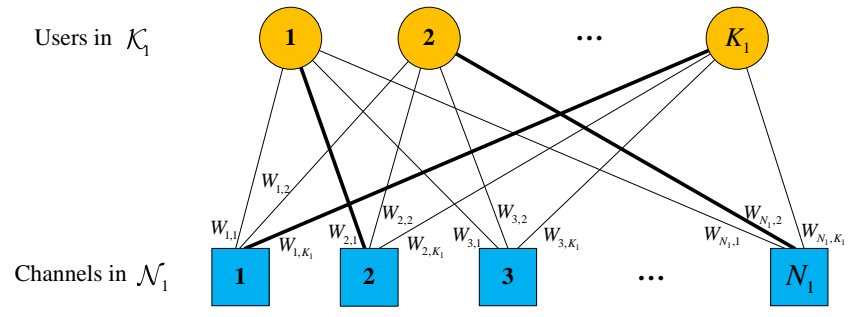

Fig. 6. Bipartite graph between the users in $\mathcal{K}_{1}$ and the channels in $\mathcal{N}_{1}$.

the objective function value. Motivated by this, the problem in (7) can be equivalently recast as the following two problems.

$$
\begin{aligned}
& \min _{\mathbf{P}_{1}, \mathbf{X}_{1}}(1-\alpha) \sum_{n_{1} \in \mathcal{N}_{1}} \sum_{k_{1} \in \mathcal{K}_{1}} p_{n_{1}, k_{1}} x_{n_{1}, k_{1}} \\
& \text { s.t. } \mathrm{C} 1:(1-\alpha) \sum_{n_{1} \in \mathcal{N}_{1}} \sum_{k_{1} \in \mathcal{K}_{1}} p_{n_{1}, k_{1}} x_{n_{1}, k_{1}} \leq P^{\mathrm{max}} \\
& \mathrm{C} 2: R_{k_{1}} \geq R_{k_{1}}^{\mathrm{req}}, \forall k_{1} \in \mathcal{K}_{1} \\
& \mathrm{C} 3: \sum_{n_{1} \in \mathcal{N}_{1}} x_{n_{1}, k_{1}}=1, \forall k_{1} \in \mathcal{K}_{1} \\
& \mathrm{C} 4: \sum_{k_{1} \in \mathcal{K}_{1}} x_{n_{1}, k_{1}} \leq 1, \forall n_{1} \in \mathcal{N}_{1} \\
& \mathrm{C} 5: x_{n_{1}, k_{1}} \in\{0,1\}, \forall n_{1} \in \mathcal{N}_{1}, \forall k_{1} \in \mathcal{K}_{1} \\
& \mathrm{C} 6: p_{n_{1}, k_{1}} \geq 0, \forall n_{1} \in \mathcal{N}_{1}, \forall k_{1} \in \mathcal{K}_{1} .
\end{aligned}
$$

$\max _{\mathbf{P}_{\mathbf{2}}, \mathbf{X}_{\mathbf{2}}} \min _{\mathrm{k}_{1} \in \mathcal{K}_{1}} E_{k_{1}}$

$$
\begin{array}{ll}
\text { s.t. } \mathrm{C} 1: \sum_{n_{2} \in \mathcal{N}_{2}} \sum_{k_{2} \in \mathcal{K}_{2}} p_{n_{2}, k_{2}} x_{n_{2}, k_{2}} \leq P^{\max }-\Theta \\
\mathrm{C} 2: R_{k_{2}} \geq R_{k_{2}}^{\text {req }}, \forall k_{2} \in \mathcal{K}_{2} \\
\mathrm{C} 3: \sum_{n_{2} \in \mathcal{N}_{2}} x_{n_{2}, k_{2}}=1, \forall k_{2} \in \mathcal{K}_{2} \\
\mathrm{C} 4: \sum_{k_{2} \in \mathcal{K}_{2}} x_{n_{2}, k_{2}} \leq 1, \forall n_{2} \in \mathcal{N}_{2} \\
\mathrm{C} 5: x_{n_{2}, k_{2}} \in\{0,1\}, \forall n_{2} \in \mathcal{N}_{2}, \forall k_{2} \in \mathcal{K}_{2} \\
\mathrm{C} 6: p_{n_{2}, k_{2}} \geq 0, \forall n_{2} \in \mathcal{N}_{2}, \forall k_{2} \in \mathcal{K}_{2},
\end{array}
$$

where $\Theta$ represents the optimal value of (8).

\section{B. Problem Solution}

To solve the problem in (8), we have the following theorem.

Theorem 1. The problem in (8) is equivalent to the optimal matching problem in the bipartite graph shown in Fig. 6, where the weight of the edge $\left(k_{1}, n_{1}\right)$ is set as

$$
W_{n_{1}, k_{1}}=\frac{\sigma^{2}}{g_{n_{1}, k_{1}}}\left(2^{\frac{R_{k_{1}}^{\mathrm{req}}}{(1-\alpha) B_{1}}}-1\right) \text {. }
$$

Proof: See Appendix A.

The optimal matching problem is a classical problem in graph theory, which can be tackled by the Kuhn-Munkras (KM) algorithm [36]. The computational complexity of the KM algorithm for solving the problem in (8) is $O\left(\left(N_{1}+K_{1}\right)^{3}\right)$.
The problem in (9) is a mixed-integer non-convex programming problem, which is very hard to tackle directly. To solve it, we first define a new set of variables $\mathbf{S}_{2}=\left\{s_{n_{2}, k_{2}} \mid n_{2} \in \mathcal{N}_{2}, \forall k_{2} \in \mathcal{K}_{2}\right\}$, where $s_{n_{2}, k_{2}}$ is defined as $s_{n_{2}, k_{2}}=p_{n_{2}, k_{2}} x_{n_{2}, k_{2}}$. Substituting $s_{n_{2}, k_{2}}$ into the problem in (9) and relaxing $x_{n_{2}, k_{2}}$ into [0,1] (i.e., $0 \leq x_{n_{2}, k_{2}} \leq 1$ ), we can get the following problem.

$$
\begin{aligned}
& \max _{\mathbf{S}_{\mathbf{2}}, \mathbf{X}_{\mathbf{2}}} \min _{\mathrm{k}_{1} \in \mathcal{K}_{1}} \alpha \sum_{n_{2} \in \mathcal{N}_{2}} \sum_{k_{2} \in \mathcal{K}_{2}} g_{n_{2}, k_{1}} s_{n_{2}, k_{2}} \\
& \text { s.t. C1 }: \sum_{n_{2} \in \mathcal{N}_{2}} \sum_{k_{2} \in \mathcal{K}_{2}} s_{n_{2}, k_{2}} \leq P^{\mathrm{max}}-\Theta \\
& \mathrm{C} 2: \sum_{n_{2} \in \mathcal{N}_{2}} x_{n_{2}, k_{2}} B_{2} \log _{2}\left(1+\frac{s_{n_{2}, k_{2}} g_{n_{2}, k_{2}}}{x_{n_{2}, k_{2}} \sigma^{2}}\right) \\
& \geq R_{k_{2}}^{\mathrm{req}}, \forall k_{2} \in \mathcal{K}_{2} \\
& \mathrm{C} 3: \sum_{n_{2} \in \mathcal{N}_{2}} x_{n_{2}, k_{2}}=1, \forall k_{2} \in \mathcal{K}_{2} \\
& \mathrm{C} 4: \sum_{k_{2} \in \mathcal{K}_{2}} x_{n_{2}, k_{2}} \leq 1, \forall n_{2} \in \mathcal{N}_{2} \\
& \mathrm{C} 5: 0 \leq x_{n_{2}, k_{2}} \leq 1, \forall n_{2} \in \mathcal{N}_{2}, \forall k_{2} \in \mathcal{K}_{2} \\
& \mathrm{C} 6: s_{n_{2}, k_{2}} \geq 0, \forall n_{2} \in \mathcal{N}_{2}, \forall k_{2} \in \mathcal{K}_{2} .
\end{aligned}
$$

The non-smoothness of the objective function in (11) makes the problem intractable. To deal with this issue, we introduce a new variable $\Delta$ into (11) and transform it into its epigraph form, which is specified as

$$
\begin{aligned}
\max _{\mathbf{S}_{2}, \mathbf{X}_{\mathbf{2}}, \Delta} & \Delta \\
\text { s.t. } \mathrm{C} 1: & \sum_{n_{2} \in \mathcal{N}_{2}} \sum_{k_{2} \in \mathcal{K}_{2}} s_{n_{2}, k_{2}} \leq P^{\mathrm{max}}-\Theta \\
\mathrm{C} 2: & \sum_{n_{2} \in \mathcal{N}_{2}} x_{n_{2}, k_{2}} B_{2} \log _{2}\left(1+\frac{s_{n_{2}, k_{2}} g_{n_{2}, k_{2}}}{x_{n_{2}, k_{2}} \sigma^{2}}\right) \\
& \geq R_{k_{2}}^{\mathrm{req}}, \forall k_{2} \in \mathcal{K}_{2} \\
\mathrm{C} 3: & \sum_{n_{2} \in \mathcal{N}_{2}} x_{n_{2}, k_{2}}=1, \forall k_{2} \in \mathcal{K}_{2} \\
\mathrm{C} 4: & \sum_{k_{2} \in \mathcal{K}_{2}} x_{n_{2}, k_{2}} \leq 1, \forall n_{2} \in \mathcal{N}_{2} \\
\mathrm{C} 5: & 0 \leq x_{n_{2}, k_{2}} \leq 1, \forall n_{2} \in \mathcal{N}_{2}, \forall k_{2} \in \mathcal{K}_{2} \\
\mathrm{C} 6: & s_{n_{2}, k_{2}} \geq 0, \forall n_{2} \in \mathcal{N}_{2}, \forall k_{2} \in \mathcal{K}_{2} \\
\mathrm{C} 7: & 0 \leq \Delta \leq \alpha \sum_{n_{2} \in \mathcal{N}_{2}} \sum_{k_{2} \in \mathcal{K}_{2}} g_{n_{2}, k_{1}} s_{n_{2}, k_{2}}, \forall k_{1} \in \mathcal{K}_{1} .
\end{aligned}
$$

For the problem in (12), we have the following theorem.

Theorem 2. The problem in (12) is jointly convex in $\mathbf{S}_{\mathbf{2}}, \mathbf{X}_{\mathbf{2}}$, and $\Delta$.

Proof: See Appendix B.

The convex problem in (12) can be solved optimally by the general convex optimization algorithms, such as the Interior Point Method [37]. However, there are many control variables included in (12) which are mutually constrained by the constraints C1-C7. As such, adopting the general algorithm to 
solve the problem in (12) will consume a lot of computational time that is intolerable in a real-time communication system. To overcome this difficulty, we analyze the special structure of the problem in (12) and adopt the Lagrangian dual decomposition technique to propose a low-complexity algorithm. Particularly, the partial Lagrangian of (12) is given by (13) [38], where $\lambda, \mu=\left\{\mu_{k_{2}} \mid k_{2} \in \mathcal{K}_{2}\right\}$, and $\nu=\left\{\nu_{k_{1}} \mid k_{1} \in \mathcal{K}_{1}\right\}$ are the dual variables corresponding to the constraints $\mathrm{C} 1, \mathrm{C} 2$, and $\mathrm{C} 7$ in (12).

$$
\begin{aligned}
& L\left(\mathbf{S}_{\mathbf{2}}, \mathbf{X}_{\mathbf{2}}, \Delta, \lambda, \mu, \nu\right) \\
& =\Delta+\lambda\left(P^{\max }-\Theta-\sum_{n_{2} \in \mathcal{N}_{2}} \sum_{k_{2} \in \mathcal{K}_{2}} s_{n_{2}, k_{2}}\right) \\
& +\sum_{k_{2} \in \mathcal{K}_{2}} \mu_{k_{2}}\left(\sum_{n_{2} \in \mathcal{N}_{2}} x_{n_{2}, k_{2}} B_{2} \log _{2}\left(1+\frac{s_{n_{2}, k_{2}} g_{n_{2}, k_{2}}}{x_{n_{2}, k_{2}} \sigma^{2}}\right)-R_{k_{2}}^{\mathrm{req}}\right) \\
& +\sum_{k_{1} \in \mathcal{K}_{1}} \nu_{k_{1}}\left(\alpha \sum_{n_{2} \in \mathcal{N}_{2}} \sum_{k_{2} \in \mathcal{K}_{2}} g_{n_{2}, k_{1}} s_{n_{2}, k_{2}}-\Delta\right)
\end{aligned}
$$

Owing to the convexity, the dual gap between the primal problem in (12) and its dual problem is zero [37]. Thus, we can tackle the primal problem by solving its dual problem. Specifically, the dual problem of (12) is given by

$$
\begin{aligned}
\min _{\lambda, \mu, \nu} \max _{\mathbf{S}_{\mathbf{2}}, \mathbf{X}_{\mathbf{2}}, \Delta} L\left(\mathbf{S}_{\mathbf{2}}, \mathbf{X}_{\mathbf{2}}, \Delta, \lambda, \mu, \nu\right) \\
\text { s.t. } \mathrm{C} 1: \sum_{n_{2} \in \mathcal{N}_{2}} x_{n_{2}, k_{2}}=1, \forall k_{2} \in \mathcal{K}_{2} \\
\mathrm{C} 2: \sum_{k_{2} \in \mathcal{K}_{2}} x_{n_{2}, k_{2}} \leq 1, \forall n_{2} \in \mathcal{N}_{2} \\
\mathrm{C} 3: 0 \leq x_{n_{2}, k_{2}} \leq 1, \forall n_{2} \in \mathcal{N}_{2}, \forall k_{2} \in \mathcal{K}_{2} \\
\mathrm{C} 4: s_{n_{2}, k_{2}} \geq 0, \forall n_{2} \in \mathcal{N}_{2}, \forall k_{2} \in \mathcal{K}_{2} .
\end{aligned}
$$

According to the dual theory [37], the objective function of (14) can be rearranged as

$$
\begin{aligned}
& \min _{\lambda, \mu, \nu} \max _{\mathbf{S}_{\mathbf{2}}, \mathbf{X}_{\mathbf{2}}, \Delta} L\left(\mathbf{S}_{\mathbf{2}}, \mathbf{X}_{\mathbf{2}}, \Delta, \lambda, \mu, \nu\right) \\
& =\min _{\lambda, \mu, \nu} \max _{\Delta} \max _{\mathbf{X}_{\mathbf{2}}} \max _{\mathbf{S}_{\mathbf{2}}} L\left(\mathbf{S}_{\mathbf{2}}, \mathbf{X}_{\mathbf{2}}, \Delta, \lambda, \mu, \nu\right) .
\end{aligned}
$$

The equation in (15) demonstrates that we can sequentially solve $\mathbf{S}_{\mathbf{2}}, \mathbf{X}_{2}, \Delta$, and the dual variables $\lambda, \mu$, and $\nu$. To obtain $s_{n_{2}, k_{2}}^{*}$, we take the partial derivative of $L\left(\mathbf{S}_{\mathbf{2}}, \mathbf{X}_{\mathbf{2}}, \Delta, \lambda, \mu, \nu\right)$ with respect to $s_{n_{2}, k_{2}}$ and get

$$
\begin{aligned}
& \frac{\partial L\left(\mathbf{S}_{\mathbf{2}}, \mathbf{X}_{\mathbf{2}}, \Delta, \lambda, \mu, \nu\right)}{\partial s_{n_{2}, k_{2}}} \\
& =\frac{B_{2} \mu_{k_{2}} g_{n_{2}, k_{2}} x_{n_{2}, k_{2}}}{\ln (2)\left(g_{n_{2}, k_{2}} s_{n_{2}, k_{2}}+\sigma^{2} x_{n_{2}, k_{2}}\right)}+\sum_{k_{1} \in \mathcal{K}_{1}} \alpha \nu_{k_{1}} g_{n_{2}, k_{1}}-\lambda .
\end{aligned}
$$

Setting the above formula equal to zero and rearranging it yield

$$
\begin{gathered}
s_{n_{2}, k_{2}}^{*}=\left[\frac{B_{2} \mu_{k_{2}} x_{n_{2}, k_{2}}}{\ln 2\left(\lambda-\sum_{k_{1} \in \mathcal{K}_{1}} \alpha \nu_{k_{1}} g_{n_{2}, k_{1}}\right)}-\frac{\sigma^{2} x_{n_{2}, k_{2}}}{g_{n_{2}, k_{2}}}\right]^{+} \\
\stackrel{(\mathrm{a})}{=} x_{n_{2}, k_{2}}\left[\frac{B_{2} \mu_{k_{2}}}{\ln 2\left(\lambda-\sum_{k_{1} \in \mathcal{K}_{1}} \alpha \nu_{k_{1}} g_{n_{2}, k_{1}}\right)}-\frac{\sigma^{2}}{g_{n_{2}, k_{2}}}\right]^{+},
\end{gathered}
$$

where $[y]^{+}=\max \{0, y\}$ and equation (a) is satisfied due to that $x_{n_{2}, k_{2}} \geq 0$.

Since $s_{n_{2}, k_{2}}=p_{n_{2}, k_{2}} x_{n_{2}, k_{2}}$, we can obtain $p_{n_{2}, k_{2}}^{*}$ according to (17), which is specified as

$$
p_{n_{2}, k_{2}}^{*}=\left[\frac{B_{2} \mu_{k_{2}}}{\ln 2\left(\lambda-\sum_{k_{1} \in \mathcal{K}_{1}} \alpha \nu_{k_{1}} g_{n_{2}, k_{1}}\right)}-\frac{\sigma^{2}}{g_{n_{2}, k_{2}}}\right]^{+} \text {. }
$$

Given $\mathbf{P}_{2}^{*}$, the dual problem of channel assignment can be transformed into

$$
\begin{aligned}
& \max _{\mathbf{X}_{2}} \sum_{k_{2} \in \mathcal{K}_{2}} \sum_{n_{2} \in \mathcal{N}_{2}} w_{n_{2}, k_{2}} x_{n_{2}, k_{2}} \\
& \text { s.t. } \mathrm{C} 1: \sum_{n_{2} \in \mathcal{N}_{2}} x_{n_{2}, k_{2}}=1, \forall k_{2} \in \mathcal{K}_{2} \\
& \mathrm{C} 2: \sum_{k_{2} \in \mathcal{K}_{2}} x_{n_{2}, k_{2}} \leq 1, \forall n_{2} \in \mathcal{N}_{2} \\
& \mathrm{C} 3: 0 \leq x_{n_{2}, k_{2}} \leq 1, \forall n_{2} \in \mathcal{N}_{2}, \forall k_{2} \in \mathcal{K}_{2},
\end{aligned}
$$

where $w_{n_{2}, k_{2}}$ is a constant as

$$
\begin{aligned}
w_{n_{2}, k_{2}}= & \mu_{k_{2}} B_{2} \log _{2}\left(1+\frac{p_{n_{2}, k_{2}}^{*} g_{n_{2}, k_{2}}}{\sigma^{2}}\right) \\
& +p_{n_{2}, k_{2}}^{*}\left(\sum_{k_{1} \in \mathcal{K}_{1}} \alpha \nu_{k_{1}} g_{n_{2}, k_{1}}-\lambda\right) .
\end{aligned}
$$

The problem in (19) is a linear programming problem (LP), as both the objective function and all constraints are linear. Solving the problem in (19) by the typical LP algorithm (e.g., cutting-plane method) will lead to the complexity of $O\left(\left(N_{2} K_{2}\right)^{3.5}\right)$ [39]. To further reduce the computational complexity, we analyze the special structure of (19) and reformulate it as a matching problem. Specifically, we have the following theorem.

Theorem 3. Among the optimal solutions of (19) (denoted by $\mathbf{X}_{2}^{*}=\left\{x_{n_{2}, k_{2}}^{*}\right\}$ ), there must exist one, where all variables are binary, i.e., $x_{n_{2}, k_{2}}^{*}=0$ or $1, \forall n_{2} \in \mathcal{N}_{2}, \forall k_{2} \in \mathcal{K}_{2}$.

Proof: Proof. See Appendix C.

According to Theorem 3, we can set all $x_{n_{2}, k_{2}}$ as binary variables without affecting the optimal solutions. As such, the problem in (19) can be descried as the one that allocating 
each user in $\mathcal{K}_{2}$ with one channel in $\mathcal{N}_{2}$ so as to maximize the linear summation of the utility, where the utility of user $k_{2}$ on channel $n_{2}$ is defined as the value in (20). As described, the problem in (19) is an optimal matching problem in graph theory [36], thereby we have the following corollary.

Corollary 1. The optimal solutions of (19) are equivalent to the optimal matching results between the users in $\mathcal{K}_{2}$ and the channels in $\mathcal{N}_{2}$, where the weigh is $w_{n_{2}, k_{2}}, \forall n_{2} \in \mathcal{N}_{2}, \forall k_{2} \in$ $\mathcal{K}_{2}$.

Remark 1. The problem in (19) is a typical LP, which may include multiple optimal solutions including binary variables or non-binary variables [40]. As such, solving the LP directly, we may get the solutions with non-binary variables, i.e., $0<x_{n_{2}, k_{2}}<1, \exists n_{2} \in \mathcal{N}_{2}, k_{2} \in \mathcal{K}_{2}$. However, the primal problem in (9) requires that all variables $x_{n_{2}, k_{2}}$ must be binary. For the non-binary variables, we should round them to 0 or 1 , which cannot guarantee the optimality of the solutions. On the contrary, if we solve (19) through the matching problem, the acquired solutions are binary and optimal ${ }^{4}$. Furthermore, the computational complexity by solving the LP is $O\left(\left(N_{2} K_{2}\right)^{3.5}\right)$ [39], while the computational complexity cased by solving the optimal matching problem is $O\left(\left(N_{2}+K_{2}\right)^{3}\right)$ [36]. Therefore, the computational complexity can be reduced significantly through the equivalent problem transformation. Furthermore, in the optimal matching problem, all constrains on $x_{n_{2}, k_{2}}$ are the same with those in (9) (i.e., the original problem), as such the obtained solutions must be correct original solutions.

After getting $\mathbf{P}_{2}^{*}$ and $\mathbf{X}_{2}^{*}$, the optimization problem for $\Delta$ can be formulated as the following problem according to (14) and the constraint $\mathrm{C} 7$ in (12).

$$
\begin{aligned}
& \max _{\Delta}\left(1-\sum_{k_{1} \in \mathcal{K}_{1}} \nu_{k_{1}}\right) \Delta \\
& \text { s.t. } 0 \leq \Delta \leq \alpha \sum_{n_{2} \in \mathcal{N}_{2}} \sum_{k_{2} \in \mathcal{K}_{2}} g_{n_{2}, k_{1}} p_{n_{2}, k_{2}}^{*} x_{n_{2}, k_{2}}^{*}, \forall k_{1} \in \mathcal{K}_{1} .
\end{aligned}
$$

For the problem in (21), we can easily acquire its optimal solution, which is denoted by $\Delta^{*}$ and specified as

$$
\Delta^{*}=\left\{\begin{array}{ll}
\Lambda, & \text { if } \sum_{k_{1} \in \mathcal{K}_{1}} \nu_{k_{1}}<1 \\
0, & \text { if } \sum_{k_{1} \in \mathcal{K}_{1}} \nu_{k_{1}} \geq 1
\end{array},\right.
$$

where

$$
\Lambda=\min _{k_{1} \in \mathcal{K}_{1}} \alpha \sum_{n_{2} \in \mathcal{N}_{2}} \sum_{k_{2} \in \mathcal{K}_{2}} g_{n_{2}, k_{1}} p_{n_{2}, k_{2}}^{*} x_{n_{2}, k_{2}}^{*} .
$$

After getting $\mathbf{P}_{2}^{*}, \mathbf{X}_{2}^{*}$, and $\Delta^{*}$, we can solve the outer optimization problem in (14) for the dual variables $\lambda, \mu$, and $\nu$. In particular, a subgradient method can be employed to obtain the optimal dual variables in an iterative manner. The iterative

\footnotetext{
${ }^{4}$ The optimal matching problem is a classical problem in graph theory [36], [41], which can be solved optimally by the KM algorithm in polynomial time. The optimality of the KM algorithm is based on the relationship between vertex labelling and perfect matching. Adopting a skilful searching method, the KM algorithm quickly evaulates all of the feasible matching results and finds the optimal one from them. For a more detailed explanation, please see the Theorem 5.5 in [41].
}

formulas are given by (24), (25), and (26), where $t$ denotes the iteration time, and $\tau_{\lambda}^{t}, \tau_{\nu_{k_{1}}}^{t}$, and $\tau_{\mu_{k_{2}}}^{t}$ represent the step sizes in $t$-th iteration, which can be set as square summable but not summable values.

\section{The Overall Algorithm}

The overall algorithm, referred to as joint power-andchannel allocation algorithm (JPCA), is summarized in Algorithm 1. The main procedure of the JPCA is sequentially solving the problems in (8) and (9). Specifically, the problem in (8) is recast as an optimal matching problem and solved by the KM algorithm. Then, the objective value of (8) is substituted into (9). Afterward, the problem in (9) is solved by an iterative algorithm, which is designed based on the Lagrangian dual decomposition technique. In what follows, we analyze the convergence and complexity of the proposed algorithm.

Theorem 4. The proposed JPCA can converge to the optimal value of (12).

Proof: See Appendix D.

The feasible region of the problem in (12) is larger than that of the problem in (9), such that the optimal value of (12) achieves the upper bound of the objective function in (9). On the other hand, the solutions obtained by the JPCA satisfy all of the constraints in (9), that is, they are also the feasible solutions of (9). According to Theorem 4, we know that the optimal value of (12) must be no larger than the maximum objective function value of (9). Therefore, the problems in (9) and (12) have the same optimal value, and the JPCA can get their optimal solutions.

The complexity of the JPCA is dominated by steps 3, 5,6 , and 8 . In step 3 , the optimal matching problem can be solved by the KM algorithm, the complexity of which is $O\left(\left(N_{1}+K_{1}\right)^{3}\right)$. In each iteration (i.e., steps 4-11), $N_{2} K_{2}$ power allocation variables and $1+K_{1}+K_{2}$ dual variables should be calculated in steps 5 and 8, which leads to the complexity of $O\left(N_{2} K_{2}+1+K_{1}+K_{2}\right)$. In step 6, the optimal matching problem can also be solved by the KM algorithm, as a consequence its complexity is $O\left(\left(N_{2}+K_{2}\right)^{3}\right)$. Besides, it is indicated in [42] that the subgradient method can converge to the desired state only after $O\left(\frac{1}{\varepsilon^{2}}\right)$ iterations. Therefore, the total complexity of the JPCA is $O\left(\left(N_{1}+K_{1}\right)^{3}+\left(\frac{1}{\varepsilon^{2}}\right)\left(\left(N_{1}+K_{1}\right)^{3}+\left(N_{2} K_{2}+1+K_{1}+K_{2}\right)\right)\right)$. In the condition that $K_{1}, K_{2}, N_{1}, N_{2} \gg 1$ and $\varepsilon$ is small enough, the total complexity of Algorithm 1 can be approximately equal to $O\left(\left(\frac{1}{\varepsilon^{2}}\right)\left(N_{1}+K_{1}\right)^{3}\right)$.

\section{Simulation Result}

In this section, we present abundant simulation results to investigate the performance of our designed dual-band SWIPT network and the proposed algorithm. The detailed simulation parameters are summarized in Table II, where the covering radius of the HSR is set according to a general power harvester with $-30 \mathrm{dBm}$ receiver sensitivity [3], and $B_{1}$ is 8 times of $B_{2}$ corresponding to their carrier frequencies. To illustrate 


$$
\begin{gathered}
\lambda^{t+1}=\left[\lambda^{t}-\tau_{\lambda}^{t}\left(P^{\max }-\Theta-\sum_{n_{2} \in \mathcal{N}_{2}} \sum_{k_{2} \in \mathcal{K}_{2}} p_{n_{2}, k_{2}}^{*} x_{n_{2}, k_{2}}^{*}\right)\right]^{+}, \\
\nu_{k_{1}}^{t+1}=\left[\nu_{k_{1}}^{t}-\tau_{\nu_{k_{1}}}^{t}\left(\alpha \sum_{n_{2} \in \mathcal{N}_{2}} \sum_{k_{2} \in \mathcal{K}_{2}} g_{n_{2}, k_{1}} p_{n_{2}, k_{2}}^{*} x_{n_{2}, k_{2}}^{*}-\Delta^{*}\right)\right]^{+}, \\
\mu_{k_{2}}^{t+1}=\left[\mu_{k_{2}}^{t}-\tau_{\mu_{k_{2}}}^{t}\left(\sum_{n_{2} \in \mathcal{N}_{2}} x_{n_{2}, k_{2}}^{*} B_{2} \log _{2}\left(1+\frac{p_{n_{2}, k_{2}}^{*} g_{n_{2}, k_{2}}}{\sigma^{2}}\right)-R_{k_{2}}^{\mathrm{req}}\right)\right]^{+},
\end{gathered}
$$

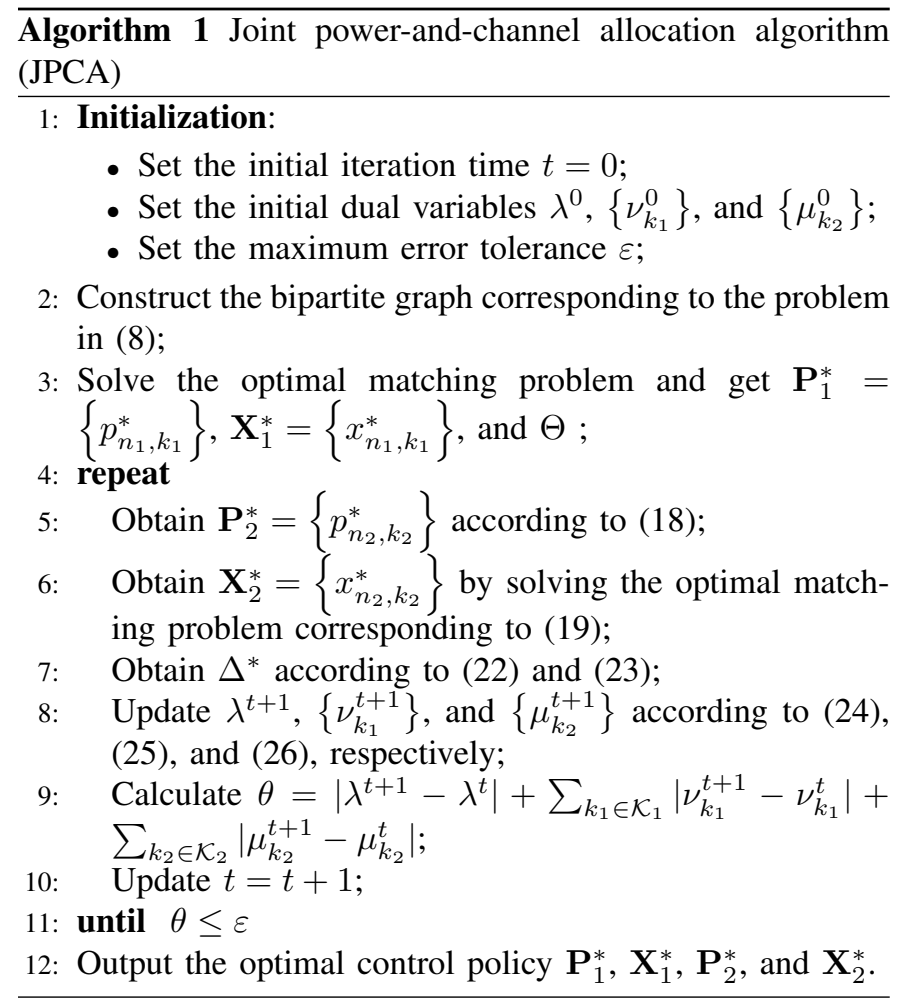

the advantages of the designed network, we compare it with the single-band networks with only LF band or HF band. For fairness, the resource allocation algorithms applied in the single-band networks are the same with our proposed algorithm. On the other hand, to demonstrate the benefits through optimizing the resource allocation, we compare our algorithm with the random scheme under the same network architecture. In the random scheme, the channels are randomly assigned to the users with equal transmission power.

Fig. 7 plots the convergence curves of the JPCA, where each curve is acquired through a random simulation realization. To simplify the simulations, the rate requirements of all users in the HSR (i.e., $R_{k_{1}}^{\text {req }}, \forall k_{1}$ ) are set as the same value. This figure shows that the minimum harvested energy, i.e., the objective function value of the problem in (7), increases after each iteration until reaching a stable state. As can be seen, the number of iterations that the algorithm converges to the stable states is usually smaller than 20 and independent on
TABLE II

SIMULATION PARAMETERS

\begin{tabular}{|c|c|}
\hline Covering radius of the WCR & $500 \mathrm{~m} \mathrm{[43]}$ \\
\hline Covering radius of the HSR & $50 \mathrm{~m}$ \\
\hline Large-scale fading & Given in (2) \\
\hline Small-scale fading & Rayleigh with 1 variance [44] \\
\hline Noise power spectrum density & $-174 \mathrm{dBm} / \mathrm{Hz}[45]$ \\
\hline Maximum transmission power, $P$ max & $40 \mathrm{Watt}[45]$ \\
\hline Subchannel bandwidth in LF band, $B_{2}$ & $180 \mathrm{KHz}[46]$ \\
\hline Subchannel bandwidth in HF band, $B_{1}$ & $1.44 \mathrm{MHz}$ \\
\hline Carrier frequency in LF band & $3.5 \mathrm{GHz}$ \\
\hline Carrier frequency in HF band & $28 \mathrm{GHz}$ \\
\hline Channel number in LF band & 100 \\
\hline Channel number in HF band & 100 \\
\hline Antenna gain & $12 \mathrm{dBi}[10]$ \\
\hline Simulation times & 5000 \\
\hline
\end{tabular}

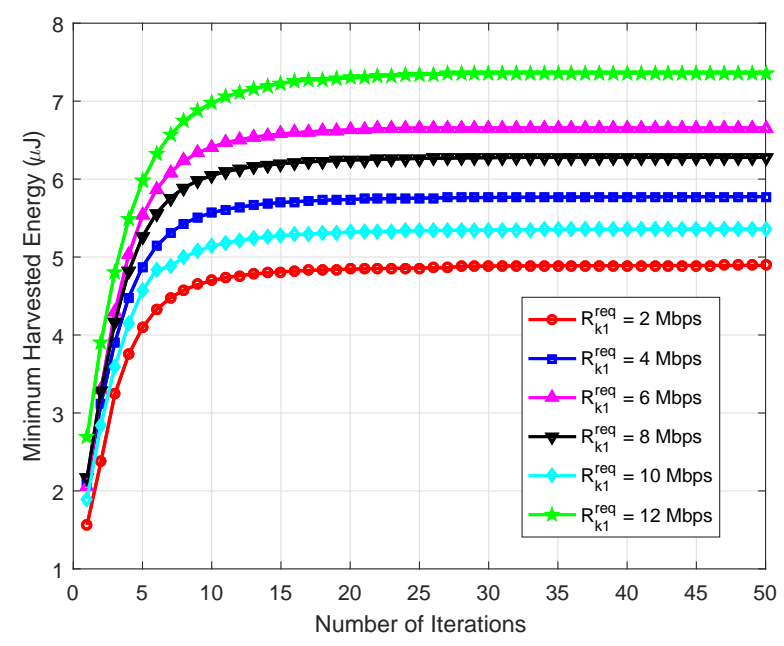

Fig. 7. Convergence evolution of the JPCA $\left(K_{1}=30, K_{2}=50, R_{k_{2}}^{\text {req }}=1\right.$ Mbps, and $\alpha=0.5$ ).

the rate requirements. Therefore, our proposed algorithm has the feature of good convergence, which is beneficial for its implementation in practical systems.

Fig. 8a shows the minimum harvested energy (MHE) versus the number of users in the HSR. It can be observed from this figure that the MHE decreases with the users in the HSR. This is because the transmission power consumed by the users in the HSR increases with the increment of the 


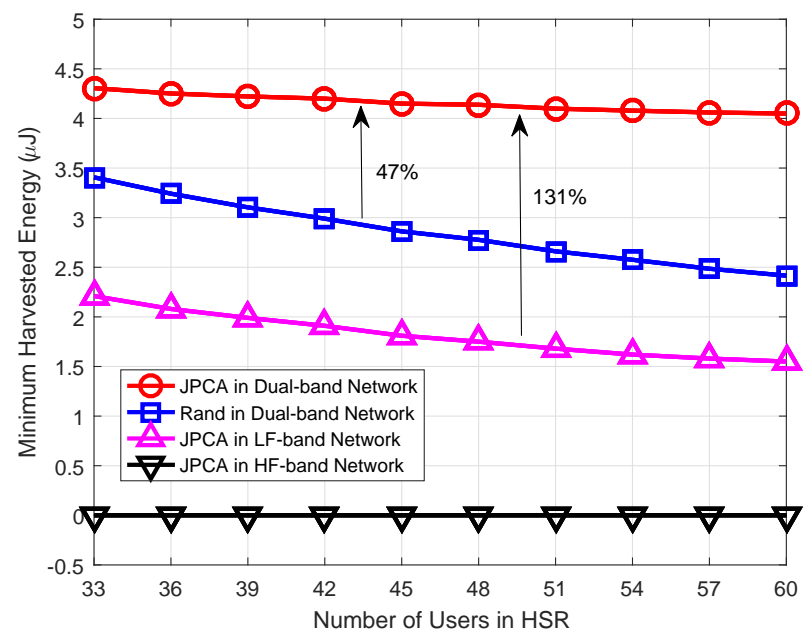

(a) Minimum harvested energy.

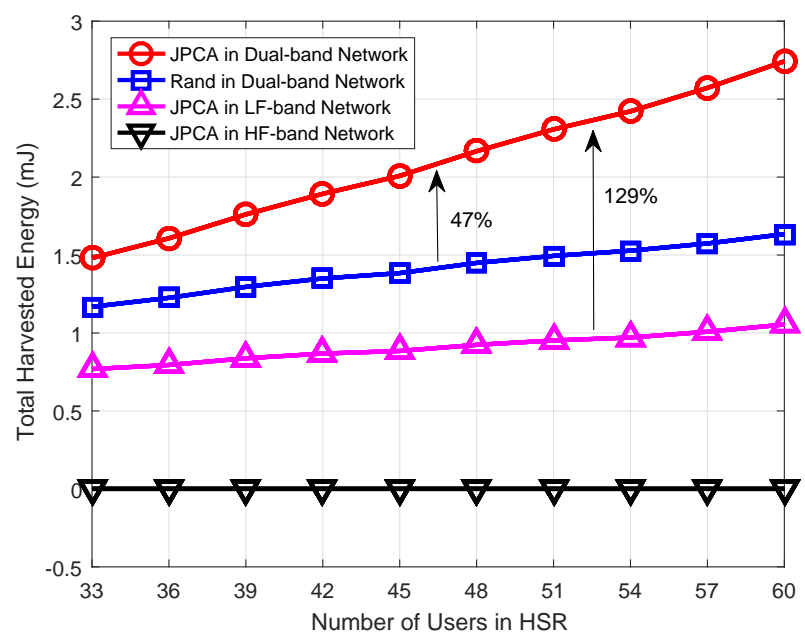

(b) Total harvested energy.

Fig. 8. Harvested energy versus the number of users in the HSR $\left(K_{2}=50\right.$, $R_{k_{1}}^{\text {req }}=2.8 \mathrm{Mbps}, R_{k_{2}}^{\text {req }}=1 \mathrm{Mbps}$, and $\alpha=0.5$ ).

users. As a consequence, the power remains for energy transfer will decrease. Furthermore, we can find that our designed network can greatly improve the harvested energy with respect to the single-band network even with the random resource allocation scheme. If the the resource allocation is optimized, the harvested energy can be further enhanced significantly. The simulation results indicate that the MHE of the users in our designed network and with our proposed algorithm can be greatly improved, that is, our scheme is capable of achieving a better fairness among users in terms of energy harvesting.

Fig. $8 \mathrm{~b}$ illustrates the total harvested energy (THE) versus the number of users in the HSR. Different from the variation tendency of the MHE shown in Fig. 8a, the THE increases with the number of users in the HSR. As aforementioned, with the increment of the users in the HSR, the energy harvested by each user will decrease. However, the users that can harvest energy increase, as a result that the THE is still improved. From the perspective of total harvested energy, our designed network incorporated with the proposed algorithm can acquire

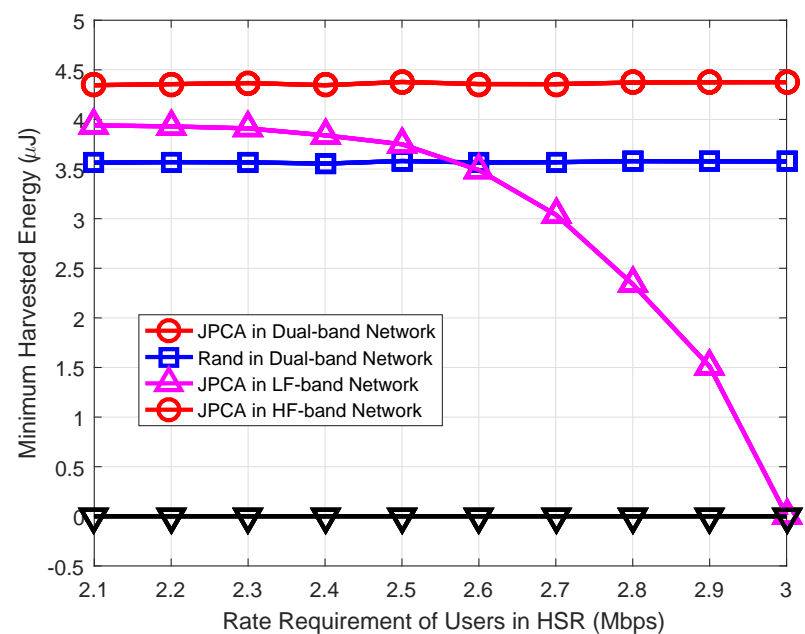

(a) Minimum harvested energy.

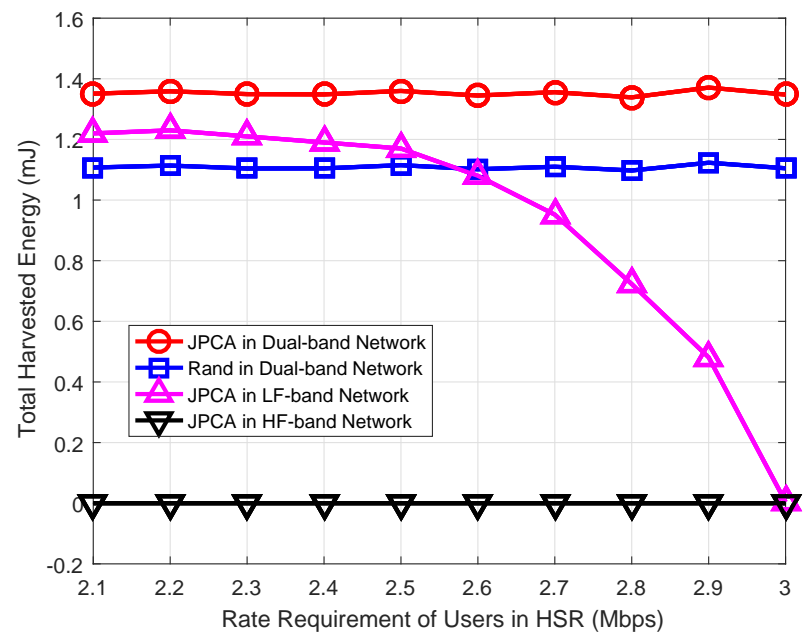

(b) Total harvested energy.

Fig. 9. Harvested energy versus the rate requirement of users in the HSR $\left(K_{1}=30, K_{2}=50, R_{k_{2}}^{\mathrm{req}}=1 \mathrm{Mbps}\right.$, and $\left.\alpha=0.5\right)$.

a great of performance gain in comparison with other schemes. In addition, the network with full HF band exhibits the worst performance, which reflects that the HF band without welldesigned directional antenna is not suitable for wireless power transfer.

Fig. 9a shows the MHE versus the rate requirement of the users in the HSR. From the simulation results, we can observe that the LF-band network is sensitive to the rate requirement of the users in the HSR, while the other schemes are insensitive. The reason is that the bandwidth in the LF band is insufficient with respect to that in the HF band. To meet the upgraded rate requirement, the transmission power allocated for the users in the HSR must be greatly elevated in the LF-band network. As a consequence, the power remained for energy transfer diminishes. Form this figure, we can conclude that the HF band is suitable for information delivery, while the LF band is suitable for energy transfer. Our designed network can fully exploit the advantages of the hybrid bands and thus achieves a good performance. 


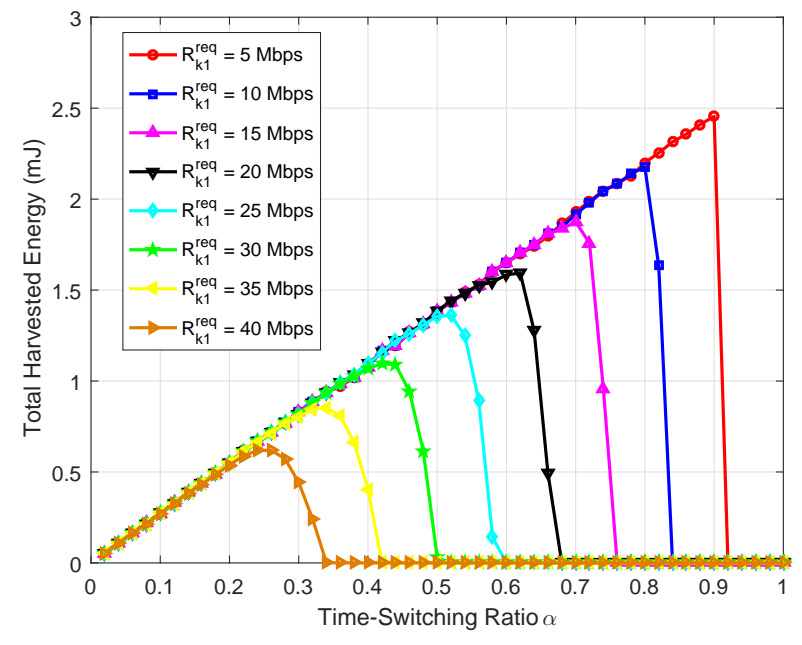

Fig. 10. The effect of the time switching ratio $\alpha$ on the total harvested energy ( $K_{1}=30, K_{2}=50$, and $\left.R_{k_{2}}^{\text {req }}=2 \mathrm{Mbps}\right)$.

Fig. 9b demonstrates the THE versus the rate requirement of the users in the HSR. This figure shows that when the rate requirement is low, the LF-band network has almost the same performance with our scheme. This result indicates that optimizing the resource allocation is more useful for energy transfer under the low rate requirement. However, with the increment of the rate requirement, the gap between the LF-band network and the dual-band network increases gradually. This is because the network architecture limits the performance gain derived by the the resource allocation algorithm. In this case, the network with hybrid bands exhibits its advantages. Therefore, in order to accommodate the dynamic of the system, both the network architecture and the resource allocation should be carefully designed.

Fig. 10 shows the effect of the time-switching ratio $\alpha$ on the THE of the users in $\mathcal{K}_{1}$. When $\alpha$ is large, more time will be used for energy harvesting, and thereby less time remains for information decoding. In order to meet the basic rate requirement of the users, more power will be consumed for the information transmission of the users in the HSR. Thus, the relationship between the THE and $\alpha$ is not linear. In fact, there is a tradeoff between the energy transfer and information delivery under the total power constraint. As depicted in Fig. 10, the THE increases first and then declines with the increment of $\alpha$. To achieve the maximum THE, there exists an optimal $\alpha$ under different system parameters. This figure shows that the smaller the rate requirement, the larger the optimal $\alpha$. However, the smaller the rate requirement, the faster the descent rate of the THE. This indicates that the system becomes instability around the optimal $\alpha$ especially when the rate requirement is small. The simulation results demonstrate that the value of $\alpha$ for achieving a better rateenergy tradeoff should be carefully selected according to different system parameters.

\section{CONCLUSION}

In this paper, we have investigated the SWIPT network design and optimization problems by jointly considering $5 \mathrm{G}$ LF and HF channels. Specifically, we have first measured the large-scale fading at $3.5 \mathrm{GHz}$ and $28 \mathrm{GHz}$ in both outdoor and outdoor-to-indoor scenarios. We have compared the propagation properties of these two channels and constructed a more accurate channel model. Based on the measurement results, we have designed a dual-band network to facilitate the implementation of the SWIPT technique. Furthermore, we have optimized the power allocation and channel assignment for all users in the network in order to enhance the energyharvesting efficiency. Finally, we have compared our proposed network and algorithm with other schemes via simulations. The simulation results have verified that the dual-band network can improve the energy-harvesting efficiency with respect to the single-band network, and the performance of the dualband network can be further boosted significantly through optimizing the resource allocation.

\section{APPENDIX A \\ PROOF OF THEOREM 1}

The objective function in (8) is the same with the lefthand side of the constraint $\mathrm{C}$. If the optimal value under the constraints $\mathrm{C} 2-\mathrm{C} 6$ is no larger than $P^{\max }$, the constraint $\mathrm{C} 1$ is satisfied correspondingly, otherwise it indicates that the problem in (8) is infeasible. Therefore, we can delete the constraint $\mathrm{C} 1$ from the problem in (8), which has no effect on the optimal solutions. Besides, the objective of (8) is to minimize the total transmission power. To achieve this goal, the transmission power allocated for each user should be minimized. The constraint C3 indicates that each user can only be assigned with one channel. Given the channel assignment scheme, we can easily obtain the minimum transmission power for each user according to the constraint C2 in (8). More specifically, if $x_{n_{1}, k_{1}}=1$, the the minimum transmission power can be calculated by

$$
W_{n_{1}, k_{1}}=\frac{\sigma^{2}}{g_{n_{1}, k_{1}}}\left(2^{\frac{R_{k_{1}}^{\mathrm{req}}}{(1-\alpha) B_{1}}}-1\right)
$$

As such, we can simplify the problem in (8) by replacing $p_{n_{1}, k_{1}}$ by $W_{n_{1}, k_{1}}$, such that the problem is transformed into the following channel assignment problem.

$$
\begin{aligned}
\min _{\mathbf{X}_{1}} & \sum_{n_{1} \in \mathcal{N}_{1}} \sum_{k_{1} \in \mathcal{K}_{1}} W_{n_{1}, k_{1}} x_{n_{1}, k_{1}} \\
\text { s.t. } & \text { C3 }: \sum_{n_{1} \in \mathcal{N}_{1}} x_{n_{1}, k_{1}}=1, \forall k_{1} \in \mathcal{K}_{1} \\
& \text { C4 }: \sum_{k_{1} \in \mathcal{K}_{1}} x_{n_{1}, k_{1}} \leq 1, \forall n_{1} \in \mathcal{N}_{1} \\
\text { C5 }: & x_{n_{1}, k_{1}} \in\{0,1\}, \forall n_{1} \in \mathcal{N}_{1}, \forall k_{1} \in \mathcal{K}_{1} .
\end{aligned}
$$

As can be seen, the above problem is a typical optimal matching problem in graph theory [36]. To this end, we have proofed Theorem 1. 


\section{B Proof of Theorem 2}

Since the objective function and the constraints $\mathrm{C} 1, \mathrm{C} 3-\mathrm{C} 7$ in (12) are linear, we need only to prove the convexity of the constraint $\mathrm{C} 2$.

For simplicity, we denote $R_{n_{2}, k_{2}}\left(x_{n_{2}, k_{2}}, s_{n_{2}, k_{2}}\right)=$ $x_{n_{2}, k_{2}} B_{2} \log _{2}\left(1+\frac{s_{n_{2}, k_{2}} g_{n_{2}, k_{2}}}{x_{n_{2}, k_{2}} \sigma^{2}}\right)$ and define

$$
\begin{aligned}
& R_{n_{2}, k_{2}}\left(x_{n_{2}, k_{2}}, s_{n_{2}, k_{2}}\right) \\
& =\left\{\begin{array}{cl}
x_{n_{2}, k_{2}} B_{2} \log _{2}\left(1+\frac{s_{n_{2}, k_{2}} g_{n_{2}, k_{2}}}{x_{n_{2}, k_{2}} \sigma^{2}}\right), & \text { if } 0<x_{n_{2}, k_{2}} \leq 1 \\
0 & \text { if } x_{n_{2}, k_{2}}=0
\end{array} .\right.
\end{aligned}
$$

In the following, we first prove that $R_{n_{2}, k_{2}}\left(x_{n_{2}, k_{2}}, s_{n_{2}, k_{2}}\right)$ is jointly concave in $x_{n_{2}, k_{2}}$ and $s_{n_{2}, k_{2}}$. According to the definition of concave function, we need to confir$\mathrm{m} R_{n_{2}, k_{2}}\left(x_{n_{2}, k_{2}}^{0}, s_{n_{2}, k_{2}}^{0}\right) \geq \rho R_{n_{2}, k_{2}}\left(x_{n_{2}, k_{2}}^{1}, s_{n_{2}, k_{2}}^{1}\right)+$ $(1-\rho) R_{n_{2}, k_{2}}\left(x_{n_{2}, k_{2}}^{2}, s_{n_{2}, k_{2}}^{2}\right)$ for any $x_{n_{2}, k_{2}}^{1}, x_{n_{2}, k_{2}}^{2} \in[0,1]$, $s_{n_{2}, k_{2}}^{1}, s_{n_{2}, k_{2}}^{2} \geq 0$, and $0 \leq \rho \leq 1$, where $x_{n_{2}, k_{2}}^{0}=$ $\rho x_{n_{2}, k_{2}}^{1}+(1-\rho) x_{n_{2}, k_{2}}^{2}$ and $s_{n_{2}, k_{2}}^{0}=\rho s_{n_{2}, k_{2}}^{1}+(1-\rho) s_{n_{2}, k_{2}}^{2}$. Then, we prove this conclusion in four cases.

1) $0<x_{n_{2}, k_{2}}^{1} \leq 1$ and $0<x_{n_{2}, k_{2}}^{2} \leq 1$ : In this case, $R_{n_{2}, k_{2}}\left(x_{n_{2}, k_{2}}, s_{n_{2}, k_{2}}\right)$ is the perspective function of $B_{2} \log _{2}\left(1+\frac{s_{n_{2}, k_{2}} g_{n_{2}, k_{2}}}{\sigma^{2}}\right)$. The convex optimization theory [37] indicates that the perspective function of a concave function is still concave. Since $B_{2} \log _{2}\left(1+\frac{s_{n_{2}, k_{2}} g_{n_{2}, k_{2}}}{\sigma^{2}}\right)$ is a concave function, $R_{n_{2}, k_{2}}\left(x_{n_{2}, k_{2}}, s_{n_{2}, k_{2}}\right)$ is thus concave as well.

$$
\begin{aligned}
& \text { 2) } x_{n_{2}, k_{2}}^{1}=0 \text { and } x_{n_{2}, k_{2}}^{2}=0 \text { : In this case, we can get } \\
& R_{n_{2}, k_{2}}\left(x_{n_{2}, k_{2}}^{0}, s_{n_{2}, k_{2}}^{0}\right) \\
& =R_{n_{2}, k_{2}}\left(0, s_{n_{2}, k_{2}}^{0}\right) \\
& =0 \\
& =\rho R_{n_{2}, k_{2}}\left(x_{n_{2}, k_{2}}^{1}, s_{n_{2}, k_{2}}^{1}\right)+(1-\rho) R_{n_{2}, k_{2}}\left(x_{n_{2}, k_{2}}^{2}, s_{n_{2}, k_{2}}^{2}\right) .
\end{aligned}
$$
that

3) $x_{n_{2}, k_{2}}^{1}=0$ and $0<x_{n_{2}, k_{2}}^{2} \leq 1$ : Now, it can be obtained

$$
\begin{aligned}
& R_{n_{2}, k_{2}}\left(x_{n_{2}, k_{2}}^{0}, s_{n_{2}, k_{2}}^{0}\right) \\
& =R_{n_{2}, k_{2}}\left((1-\rho) x_{n_{2}, k_{2}}^{2}, s_{n_{2}, k_{2}}^{0}\right) \\
& =(1-\rho) x_{n_{2}, k_{2}}^{2} B_{2} \log _{2}\left(1+\frac{\rho s_{n_{2}, k_{2}}^{1} g_{n_{2}, k_{2}}}{(1-\rho) x_{n_{2}, k_{2}}^{2} \sigma^{2}}+\frac{s_{n_{2}, k_{2}}^{2} g_{n_{2}, k_{2}}}{x_{n_{2}, k_{2}}^{2} \sigma^{2}}\right) \\
& \geq(1-\rho) x_{n_{2}, k_{2}}^{2} B_{2} \log _{2}\left(1+\frac{s_{n_{2}, k_{2}}^{2} g_{n_{2}, k_{2}}}{x_{n_{2}, k_{2}}^{2} \sigma^{2}}\right) \\
& =\rho R_{n_{2}, k_{2}}\left(x_{n_{2}, k_{2}}^{1}, s_{n_{2}, k_{2}}^{1}\right)+(1-\rho) R_{n_{2}, k_{2}}\left(x_{n_{2}, k_{2}}^{2}, s_{n_{2}, k_{2}}^{2}\right) .
\end{aligned}
$$

4) $0<x_{n_{2}, k_{2}}^{1} \leq 1$ and $x_{n_{2}, k_{2}}^{2}=0$ : This case is similar with $3)$, such that we can prove

$$
\begin{aligned}
& R_{n_{2}, k_{2}}\left(x_{n_{2}, k_{2}}^{0}, s_{n_{2}, k_{2}}^{0}\right) \\
& \geq \rho R_{n_{2}, k_{2}}\left(x_{n_{2}, k_{2}}^{1}, s_{n_{2}, k_{2}}^{1}\right)+(1-\rho) R_{n_{2}, k_{2}}\left(x_{n_{2}, k_{2}}^{2}, s_{n_{2}, k_{2}}^{2}\right) .
\end{aligned}
$$

To summarize, $R_{n_{2}, k_{2}}\left(x_{n_{2}, k_{2}}, s_{n_{2}, k_{2}}\right)$ is a concave function. $\sum_{n_{2} \in \mathcal{N}_{2}} x_{n_{2}, k_{2}} B_{2} \log _{2}\left(1+\frac{s_{n_{2}, k_{2}} g_{n_{2}, k_{2}}}{x_{n_{2}, k_{2}} \sigma^{2}}\right)$ is the linear summation over some concave functions and thereby is also a concave function. Furthermore, the superlevel set of a concave function is convex. Therefore, the constraint $\mathrm{C} 2$ in (12) is a convex constraint, and hence the problem in (12) is a convex problem.

\section{Proof of Theorem 3}

The problem in (19) is a typical LP. Besides, all constraints in (19) are affine, and hence the feasible region of the problem is a polytope. For the LP with a polytope-type feasible region, there must exist one optimal solution at the extreme point of the ploytope. Therefore, to prove Theorem 3, we need only to prove that all variables corresponding to the extreme points of the polytope (i.e., $\mathbf{X}_{2}=\left\{x_{n_{2}, k_{2}}\right\}$ ) are binary. Firstly, we present the definition of the extreme point.

Definition 1. (Extreme point [40]): An extreme point $\mathbf{X}^{\varepsilon}$ of a convex set $C$ is a point, belonging to its closure $\bar{C}$, which cannot be expressible as a convex combination of the points in $\bar{C}$ distinct from $\mathbf{X}^{\varepsilon}$, that is, for $\mathbf{X}^{\varepsilon} \in \bar{C}$ and any $\mathbf{X}^{\prime}, \mathbf{X}^{\prime \prime} \in$ $\bar{C} \backslash \mathbf{X}^{\varepsilon}, u \mathbf{X}^{\prime}+(1-u) \mathbf{X}^{\prime \prime} \neq \mathbf{X}^{\varepsilon}, \forall u \in[0,1]$.

In what follows, we introduce the detailed proof by contradiction. Assume $\mathbf{X}^{\varepsilon}$ is an extreme point in the polytope, where some variables are non-binary. Without of generality, we focus on one of the non-binary variables, which is denoted by $x_{\widehat{n}_{2}, \widehat{k}_{2}}\left(0<x_{\widehat{n}_{2}, \widehat{k}_{2}}<1\right)$. To meet the constraint $\mathrm{C} 1$ in (19), i.e., $\sum_{n_{2} \in \mathcal{N}_{2}} x_{n_{2}, k_{2}}=1$, there must be at least another nonbinary variable, which is denoted by $x_{\bar{n}_{2}, \widehat{k}_{2}}\left(0<x_{\bar{n}_{2}, \widehat{k}_{2}}<1\right)$. Then, we prove our conclusion in two cases.

1) All constraints in $\mathrm{C} 2$ are strictly inequal, i.e., $\sum_{k_{2} \in \mathcal{K}_{2}} x_{n_{2}, k_{2}}<1, \forall n_{2} \in \mathcal{N}_{2}$.

In this case, we can always find another two points as

$$
\begin{aligned}
\mathbf{X}^{\prime} & =\left(\cdots, x_{\widehat{n}_{2}, \widehat{k}_{2}}+\delta, \cdots, x_{\bar{n}_{2}, \widehat{k}_{2}}-\delta, \cdots\right), \\
\mathbf{X}^{\prime \prime} & =\left(\cdots, x_{\widehat{n}_{2}, \widehat{k}_{2}}-\delta, \cdots, x_{\bar{n}_{2}, \widehat{k}_{2}}+\delta, \cdots\right),
\end{aligned}
$$

where $\delta$ is a small enough positive number and $\cdots$ denote the variables identical with those in $\mathbf{X}^{\varepsilon}$.

For $\mathbf{X}^{\prime}$, we can obtain that

$$
\begin{gathered}
\sum_{n_{2} \in \mathcal{N}_{2}} x_{n_{2}, k_{2}}^{\prime}=\sum_{n_{2} \in \mathcal{N}_{2}} x_{n_{2}, k_{2}}^{\varepsilon}=1, \\
\sum_{k_{2} \in \mathcal{K}_{2}} x_{n_{2}, k_{2}}^{\prime}= \begin{cases}\sum_{k_{2} \in \mathcal{K}_{2}} x_{n_{2}, k_{2}}^{\varepsilon}+\delta, & \text { if } n_{2}=\widehat{\mathcal{K}}_{2} . \\
\sum_{k_{2} \in \mathcal{K}_{2}} x_{n_{2}, k_{2}}^{\varepsilon}-\delta, & \text { if } n_{2}=\bar{n}_{2} \\
\sum_{k_{2} \in \mathcal{K}_{2}} x_{n_{2}, k_{2}}^{\varepsilon}, & \text { otherwise }\end{cases}
\end{gathered}
$$

Since $\delta$ is a small enough positive number, $\sum_{k_{2} \in \mathcal{K}_{2}} x_{n_{2}, k_{2}}^{\prime} \leq 1$ is satisfied. Besides, each $x_{n_{2}, k_{2}}^{\prime} \in \mathbf{X}^{\prime}$ belongs to $[0,1]$. Thus, $\mathbf{X}^{\prime}$ satisfies all constraints in (19), this is, it is a feasible solution of (19). Similarly, we can prove that $\mathbf{X}^{\prime \prime}$ is also a feasible solution of (19). However, $\mathbf{X}^{\varepsilon}=0.5 \mathbf{X}^{\prime}+0.5 \mathbf{X}^{\prime \prime}$, which contradicts the assumption that $\mathbf{X}^{\varepsilon}$ is an extreme point. 


$$
\begin{aligned}
& \mathbf{X}^{\prime}=\left(\cdots, x_{\widehat{n}_{2}, \widehat{k}_{2}}+\delta, \cdots, x_{\bar{n}_{2}, \widehat{k}_{2}}-\delta, \cdots, x_{\widehat{n}_{2}, \bar{k}_{2}}-\delta, \cdots, x_{\bar{n}_{2}, \bar{k}_{2}}+\delta, \cdots\right) \\
& \mathbf{X}^{\prime \prime}=\left(\cdots, x_{\widehat{n}_{2}, \widehat{k}_{2}}-\delta, \cdots, x_{\bar{n}_{2}, \widehat{k}_{2}}+\delta, \cdots, x_{\widehat{n}_{2}, \bar{k}_{2}}+\delta, \cdots, x_{\bar{n}_{2}, \bar{k}_{2}}-\delta, \cdots\right)
\end{aligned}
$$

2) Some of constraints in $\mathrm{C} 2$ are tight with $\mathbf{X}^{\varepsilon}$, i.e., $\sum_{k_{2} \in \mathcal{K}_{2}} x_{n_{2}, k_{2}}=1, \exists n_{2} \in \mathcal{N}_{2}$.

To meet the equal constraints in $\mathrm{C} 1$ and $\mathrm{C} 2$, there must be at least another two non-binary variables, which are denoted by $x_{\widehat{n}_{2}, \bar{k}_{2}}$ and $x_{\bar{n}_{2}, \bar{k}_{2}}$. Then, we can find another two points as (37) and (38).

For $\mathbf{X}^{\prime}$, it can be verified that

$$
\begin{aligned}
\sum_{n_{2} \in \mathcal{N}_{2}} x_{n_{2}, k_{2}}^{\prime} & =\sum_{n_{2} \in \mathcal{N}_{2}} x_{n_{2}, k_{2}}^{\varepsilon}=1, \forall k_{2} \in \mathcal{K}_{2} . \\
\sum_{k_{2} \in \mathcal{K}_{2}} x_{n_{2}, k_{2}}^{\prime} & =\sum_{k_{2} \in \mathcal{K}_{2}} x_{n_{2}, k_{2}}^{\varepsilon} \leq 1, \forall n_{2} \in \mathcal{N}_{2} .
\end{aligned}
$$

In addition, each $x_{n_{2}, k_{2}}^{\prime} \in \mathbf{X}^{\prime}$ belongs to [0,1]. As such, $\mathbf{X}^{\prime}$ is a feasible solution of (19). Similarly, $\mathbf{X}^{\prime \prime}$ is also a feasible solution of (19). Since $\mathbf{X}^{\varepsilon}$ can be expressed as $0.5 \mathbf{X}^{\prime}+0.5 \mathbf{X}^{\prime \prime}$, $\mathbf{X}^{\varepsilon}$ cannot be an extreme point.

To summarize, we can conclude that $\mathbf{X}^{\varepsilon}$ cannot be the extreme point according to the Definition 1. In other words, all variables corresponding to the extreme points of the polytope must be binary. To this end, we have proofed Theorem 3 .

\section{PRoOF of TheOREM 4}

The problems in (8) and (19) are typical optimal matching problems in graph theory, which can be solved optimally by the Kuhn-Munkras algorithm in limited time [36]. Additionally, the equations in (18), (19), and (22) indicate that the optimal primal variables can be acquired as long as the optimal dual variables are given. Therefore, to prove the convergence of the JPCA, the key is to prove that the subgradient method adopted for the dual variables can converge to their optimal solutions.

For notational simplicity, we denote $\mathrm{z}^{*}$ as the optimal dual variables and $f^{*}$ as the optimal value of (12). In addition, we use $\mathrm{z}^{t}, g^{t}$, and $\gamma^{t}$ to represent the dual variables, subgradients, and step sizes in the $t$-iteration, respectively. Then, we can get the following inequation, where $\|x\|_{2}$ denote the 2-norm of $x$.

$$
\begin{aligned}
& \left\|z^{t+1}-z^{*}\right\|_{2}^{2} \\
& =\left\|z^{t}-\gamma^{t} g^{t}-z^{*}\right\|_{2}^{2} \\
& =\left\|z^{t}-z^{*}\right\|_{2}^{2}-2 \gamma^{t}\left(z^{t}-z^{*}\right)^{T} g^{t}+\left(\gamma^{t}\right)^{2}\left\|g^{t}\right\|_{2}^{2} \\
& \stackrel{(a)}{\leq}\left\|z^{t}-z^{*}\right\|_{2}^{2}-2 \gamma^{t}\left(f\left(z^{t}\right)-f^{*}\right)+\left(\gamma^{t}\right)^{2}\left\|g^{t}\right\|_{2}^{2},
\end{aligned}
$$

where inequation (a) holds due to the definition of subgradient, this is, the subgradient of $f$ at $x$ is define as the vector $g$ that satisfies the inequation $f(y) \geq f(x)+(y-x)^{T} g$ for any $y$ [37].
According to (24)-(26), we know that $\left\|g^{t}\right\|_{2}$ is finite, and hence we can assume $\left\|g^{t}\right\|_{2} \leq G$ in each iteration. Using the recursive method for (41), we can get

$$
\begin{aligned}
& \left\|z^{t+1}-z^{*}\right\| \\
& \leq\left\|z^{1}-z^{*}\right\|_{2}^{2}-2 \sum_{i=1}^{t} \gamma^{i}\left(f\left(z^{i}\right)-f^{*}\right)+G^{2} \sum_{i=1}^{t}\left(\gamma^{i}\right)^{2} .
\end{aligned}
$$

Defining $f_{\text {best }}^{t}=\min _{i=1, \cdots, t} f\left(z^{i}\right)$, we have

$$
\left(\sum_{i=1}^{t} \gamma^{i}\right)\left(f_{\text {best }}^{t}-f^{*}\right) \leq \sum_{i=1}^{t} \gamma^{i}\left(f\left(z^{i}\right)-f^{*}\right) \text {. }
$$

Plugging (42) into (43) and rearranging it yield

$$
f_{\text {best }}^{t}-f^{*} \leq \frac{\left\|z^{1}-z^{*}\right\|_{2}^{2}+G^{2} \sum_{i=1}^{t}\left(\gamma^{i}\right)^{2}}{2 \sum_{i=1}^{t} \gamma^{i}} .
$$

The step sizes of the dual variables are set as square summable but not summable values, i.e., $\sum_{i=1}^{\infty}\left(\gamma^{i}\right)^{2}<\infty$ and $\sum_{i=1}^{\infty} \gamma^{i}=\infty$. As $t \rightarrow \infty$, the righthand side of the above inequation becomes zero, that is, $f_{\text {best }}^{t}$ converges to $f^{*}$. Therefore, the subgradient method adopted in the paper can converge to the optimal solutions of the dual variables. Correspondingly, the proposed JPCA can converge to the optimal value of (12).

\section{REFERENCES}

[1] IMT-2020 (5G) Promotion Group: $5 G$ Vision and Requirements. White paper, May 2015.

[2] J. G. Andrews, S. Buzzi, W. Choi, S. V. Hanly, A. Lozano, A. C. K. Soong, and J. C. Zhang, "What will 5G be?" IEEE J. Sel. Areas Commun., vol. 32, no. 6, pp. 1065-1082, June 2014.

[3] X. Lu, P. Wang, D. Niyato, D. I. Kim, and Z. Han, "Wireless networks with RF energy harvesting: A contemporary survey," IEEE Commun. Surveys \& Tutorials, vol. 17, no. 2, pp. 757-789, Second quarter 2015.

[4] K. Huang and V. K. N. Lau, "Enabling wireless power transfer in cellular networks: Architecture, modeling and deployment," IEEE Trans. Wireless Commun., vol. 13, no. 2, pp. 902-912, Feb. 2014.

[5] L. Varshney, "Transporting information and energy simultaneously," in Proc. IEEE ISIT'08, Toronto, Canada, Jul. 2008, pp. 1612-1616.

[6] R. Zhang and C. K. Ho, "MIMO broadcasting for simultaneous wireless information and power transfer," IEEE Trans. Wireless Commun., vol. 12 , no. 5, pp. 1989-2001, May 2013.

[7] Z. Xun, Z. Rui, and H. C. Keong, "Wireless information and power transfer: architecture design and rate-energy tradeoff," IEEE Trans. Commun., vol. 61, no. 11, pp. 4754-4767, Nov. 2013.

[8] Z. Ding, C. Zhong, D. W. K. Ng, M. Peng, H. A. Suraweera, R. Schober, and H. V. Poor, "Application of smart antenna technologies in simultaneous wireless information and power transfer," IEEE Commun. Mag., vol. 53, no. 4, pp. 86-93, Apr. 2015.

[9] R. Morsi, D. Michalopoulos, and R. Schober, "Multiuser scheduling schemes for simultaneous wireless information and power transfer over fading channels," IEEE Trans. Commun., vol. 14, no. 4, pp. 1967-1982, Apr. 2015. 
[10] D. W. K. Ng, E. S. Lo, and R. Schober, "Wireless information and power transfer: energy effciency optimization in OFDMA systems," IEEE Trans. Wireless Commun., vol. 12, no. 12, pp. 6352-6370, Dec. 2013.

[11] X. Zhou, R. Zhang, and C. K. Ho, "Wireless information and power transfer in multiuser OFDM systems," IEEE Trans. Wireless Commun., vol. 13, no. 4, pp. 2282-2294, Apr. 2014.

[12] D. Zhai, M. Sheng, X. Wang, Y. Li, J. Song, and J. Li, "Rate and energy maximization in SCMA networks with wireless information and power transfer," IEEE Commun. Lett., vol. 20, no. 2, pp. 360-363, Feb. 2016.

[13] Y. Liu, Z. Ding, M. Elkashlan, and H. V. Poor, "Cooperative nonorthogonal multiple access with simultaneous wireless information and power transfer," IEEE J. Sel. Areas Commun., vol. 34, no. 4, pp. 938 953, Apr. 2016.

[14] Y. Huang and B. Clerckx, "Joint wireless information and power transfer for an autonomous multiple antenna relay system," IEEE Commun. Lett., vol. 19, no. 7, pp. 1113-1116, July 2015.

[15] X. Wu, W. Xu, X. Dong, H. Zhang, and X. You, "Asymptotically optimal power allocation for massive MIMO wireless powered communications," IEEE Wireless Commun. Lett., vol. 5, no. 1, pp. 100-103, Feb. 2016.

[16] J. Park and B. Clerckx, "Joint wireless information and energy transfer with reduced feedback in MIMO interference channels," IEEE J. Sel. Areas Commun., vol. 33, no. 8, pp. 1563-1577, Aug. 2015.

[17] D. T. Hoang, D. Niyato, P. Wang, D. I. Kim, and Z. Han, "Ambient backscatter: A new approach to improve network performance for RFpowered cognitive radio networks," IEEE Trans. Commun., vol. 65, no. 9, pp. 3659-3674, Sep. 2017

[18] M. E. Ahmed, D. I. Kim, and K. W. Choi, "Traffic-aware optimal spectral access in wireless powered cognitive radio networks," IEEE Trans. Mobile Comput., vol. 17, no. 3, pp. 733-745, Mar. 2018.

[19] S. Guo, C. Wang, and Y. Yang, "Joint mobile data gathering and energy provisioning in wireless rechargeable sensor networks," IEEE Trans. Mobile Comput., vol. 13, no. 12, pp. 2836-2852, Dec. 2014.

[20] T. Liu, X. Wang, and L. Zheng, "A cooperative SWIPT scheme for wirelessly powered sensor networks," IEEE Trans. Commun., vol. 65 , no. 6, pp. 2740-2752, June 2017.

[21] G. Pan, H. Lei, Y. Yuan, and Z. Ding, "Performance analysis and optimization for SWIPT wireless sensor networks," IEEE Trans. Commun., vol. 65, no. 5, pp. 2291-2302, May 2017.

[22] T. D. P. Perera, D. N. K. Jayakody, S. K. Sharma, S. Chatzinotas, and J. Li, "Simultaneous wireless information and power transfer (SWIPT): Recent advances and future challenges," IEEE Commun. Surveys \& Tutorials, vol. 20, no. 1, pp. 264-302, First quarter 2018.

[23] S. Ladan, A. B. Guntupalli, and K. Wu, "A high-efficiency $24 \mathrm{GHz}$ rectenna development towards millimeter-wave energy harvesting and wireless power transmission," IEEE Transactions on Circuits and Systems I: Regular Papers, vol. 61, no. 12, pp. 3358-3366, Dec. 2014.

[24] L. Wang, M. Elkashlan, R. W. Heath, M. D. Renzo, and K. K. Wong, "Millimeter wave power transfer and information transmission," in Proc. IEEE GLOBECOM'15, San Diego, CA, USA, Dec. 2015, pp. 1-6.

[25] T. A. Khan, A. Alkhateeb, and R. W. Heath, "Millimeter wave energy harvesting," IEEE Trans. Wireless Commun., vol. 15, no. 9, pp. 60486062, Sep. 2016.

[26] G. N. Kamga and S. Aissa, "Wireless power transfer in mmWave massive MIMO systems with/without rain attenuation," IEEE Trans. Commun., to be published, doi: 10.1109/TCOMM.2018.2799217.

[27] —, "Relay-aided energy and information transmission in mmWave massive MIMO systems," in Proc. IEEE GLOBECOM'17, Singapore, Dec. 2017, pp. 1-6.

[28] S. Biswas, S. Vuppala, and T. Ratnarajah, "On the performance of mmWave networks aided by wirelessly powered relays," IEEE Journal of Selected Topics in Signal Processing, vol. 10, no. 8, pp. 1522-1537, Dec. 2016.

[29] IMT-2020 (5G) Promotion Group: $5 G$ Wireless Technology Architecture. White paper, May 2015.

[30] R. Zhang, J. Wang, Z. Zhong, C. Li, X. Du, and M. Guizani, "Energyefficient beamforming for $3.5 \mathrm{GHz} 5 \mathrm{G}$ cellular networks based on 3D spatial channel characteristics," ELSEVIER Computer Communications, vol. 121, pp. 59-70, May 2018.

[31] R. Zhang, C. L, Z. Zhong, J. Zhao, and J. Zhou, "Cross-polarized threedimensional channel measurement and modeling for small-cell street canyon scenario," IEEE Trans. Veh. Technol., to be published, doi: 10.1109/TVT.2018.2846485.

[32] A. Goldsmith, Wireless Communications. Cambridge University Press, 2005.
[33] H. Tabassum, E. Hossain, A. Ogundipe, and D. I. Kim, "Wirelesspowered cellular networks: key challenges and solution techniques," IEEE Commun. Mag., vol. 53, no. 6, pp. 63-71, June 2015.

[34] P. H. Hsieh, C. H. Chou, and T. Chiang, "An RF energy harvester with 44.1PCE at input available power of -12 dbm," IEEE Transactions on Circuits and Systems I: Regular Papers, vol. 62, no. 6, pp. 1528-1537, June 2015.

[35] http://www.powercastco.com/products/powerharvester-receivers/.

[36] J. Bondy and U. Murty, Graph Theory. Germany: Springer, 2008

[37] S. Boyd and L. Vandenberghe, Convex Optimization. Cambridge University Press, 2004

[38] J. Du, L. Zhao, J. Feng, and X. Chu, "Computation offloading and resource allocation in mixed fog/cloud computing systems with minmax fairness guarantee," IEEE Trans. Commun., vol. 66, no. 4, pp. 1594-1608, Apr. 2018.

[39] M. Sheng, D. Zhai, X. Wang, Y. Li, Y. Shi, and J. Li, "Intelligent energy and traffic coordination for green cellular networks with hybrid energy supply," IEEE Trans. Veh. Technol., vol. 66, no. 2, pp. 1631-1646, Feb. 2017.

[40] J. Dattorro, Convex Optimization \& Euclidean Distance Geometry. Meboo, 2005.

[41] J. A. Bondy and U. S. R. Murty, Graph Theory With Applications. Elsevier Science Ltd, 1976.

[42] L. Vandenberghe, "Subgradient method," University of California, Los Angeles, Tech. Rep., 2013, [On-line]. Available: http://www.seas.ucla.edu/ vandenbe/236C/lectures/sgmethod.pdf.

[43] D. W. K. Ng, E. S. Lo, and R. Schober, "Energy-efficient resource allocation in OFDMA systems with hybrid energy harvesting base station," IEEE Trans. Wireless Commun., vol. 12, no. 7, pp. 3412-3427, Jul. 2013.

[44] D. Zhai, R. Zhang, L. Cai, B. Li, and Y. Jiang, "Energy-efficient user scheduling and power allocation for NOMA-Based wireless networks with massive IoT devices," IEEE Internet of Things Journal, vol. 5, no. 3, pp. 1857-1868, June 2018.

[45] J. Gong, S. Zhou, and Z. Niu, "Optimal power allocation for energy harvesting and power grid coexisting wireless communication systems," IEEE Trans. Commun., vol. 61, no. 7, pp. 3040-3049, Jul. 2013.

[46] D. Zhai, M. Sheng, X. Wang, Z. Sun, C. Xu, and J. Li, "Energysaving resource management for D2D and cellular coexisting networks enhanced by hybrid multiple access technologies," IEEE Trans. Wireless Commun., vol. 16, no. 4, pp. 2678-2692, Apr. 2017. 